UFIFT-HEP-94 - 8

hep-th/9407169

\title{
Calculating the Rest Tension for a Polymer of String Bits ${ }^{*}$
}

\author{
Charles B. Thorn \\ Department of Physics, University of Florida, Gainesville, FL, 32611, USA
}

\begin{abstract}
We explore the application of approximation schemes from many body physics, including the Hartree-Fock method and random phase approximation (RPA), to the problem of analyzing the low energy excitations of a polymer chain made up of bosonic string bits. We accordingly obtain an expression for the rest tension $T_{0}$ of the bosonic relativistic string in terms of the parameters characterizing the microscopic string bit dynamics. We first derive an exact connection between the string tension and a certain correlation function of the many-body string bit system. This connection is made for an arbitrary interaction potential between string bits and relies on an exact dipole sum rule. We then review an earlier calculation by Goldstone of the low energy excitations of a polymer chain using RPA. We assess the accuracy of the RPA by calculating the first order corrections. For this purpose we specialize to the unique scale invariant potential, namely an attractive delta function potential in two (transverse) dimensions. We find that the corrections are large, and discuss a method for summing the large terms. The corrections to this improved RPA are roughly $15 \%$.
\end{abstract}

* Work supported in part by the Department of Energy, contract DE-FG05-86ER-40272 


\section{Introduction}

A decade and a half ago we proposed a microscopic theory of the relativistic string. ${ }^{[1-3]}$ At that time the main motivation was to seek a connection between string theory and QCD. From today's vantage point this motivation appears wrongheaded, but the model we proposed then still holds promise for a fundamental reformulation of string theory. Some of the same ideas have reemerged in recent years in the context of large $N$ matrix models, which have been the subject of vigorous investigation. ${ }^{[4-8]}$ In $1991^{[9]}$ we sketched why the wee parton model of Ref.[3] is an attractive starting point for a fundamental formulation of string theory.

In this article we begin a serious study of the detailed relationship of the microscopic physics of such a string bit model to the low energy properties of string. Presumably, the microscopic details of the model would only be apparent at the Planck scale. For the purposes of our present analysis we imagine that the string coupling is small, i.e. the string mass scale $\sqrt{T_{0}}<<M_{\text {Planck. Even though }}$ this is probably an unrealistic assumption, it is useful because it is a limit which puts highly nontrivial constraints on the string bit model. In particular, it is a limit in which the graviton, a composite of string bits in our model, must satisfy all of the low energy theorems enjoyed by Einstein's general relativity. These constraints are guaranteed, of course, once it is demonstrated that the low energy properties of our string bit system are precisely those of the relativistic string.

The work of Ref.[3] established the microscopic/macroscopic connection between string bits and the bosonic string in the context of the Random Phase Approximation (RPA). It can be argued that the corrections to RPA can be lumped into a renormalization of the string tension, and so are not important as far as the stringy properties of the low energy theory are concerned. However the actual size of the corrections to RPA was not determined in Ref.[3]. Since the actual microscopic details of the string bits may be of eventual interest (e.g. in resolving the short distance properties of gravity), we believe that it is important to assess the validity of RPA quantitatively, and this is our goal here. Moreover, the de- 
tails of the micro/macro connection are probably essential for a proper string bit understanding of the superstring. For example, must we require an exact supersymmetry at the level of string bits, or is supersymmetry only present in the low energy theory? We don't answer that question here, but it is clearly an important one, and the answer is surely going to require a more extensive treatment than that given in Ref.[3].

In the following sections we provide this more detailed study of bosonic string bits. In Section 2, we present the dynamics of a polymer of string bits in Goldstone's formulation of the many body problem. ${ }^{[10]}$ This formulation provides an elegant presentation of various "improved" versions of perturbation theory, such as Hartree-Fock methods and RPA, in terms of Feynman diagrams. In Section 3, we use this diagrammatic method to define certain "irreducible" correlation functions, for which exact low frequency theorems can be derived. These theorems are then used to show the universal presence of harmonic low energy excitations, which reproduce the usual spectrum of the relativistic string. Thus the string tension will be related to the value a certain zero-frequency correlation function $\int d t\left\langle G\left|T x^{r}(t) x^{s}(0)\right| G\right\rangle$. This holds even for string bit interaction potentials that are not differentiable or even finite at minimum separation. A case in point is the potential $V(\mathbf{x})=-\lambda_{0} \delta(\mathbf{x})$, which has the nice property of being scale invariant in two dimensions - precisely the case for the transverse space of four-dimensional space-time. But evaluation of the relevant correlation function requires an exact solution of the string bit system, which we don't have except in the case of a harmonic oscillator string bit potential. Our string bit model involves a short range, scale invariant potential. This is where we must resort to approximations. In Section 4, we review Goldstone's application of RPA to the string bit polymer problem and then proceed to calculate the first order corrections to RPA. We find that the diagrams which have the character of "self-energy" corrections are in fact quite large. Fortunately the structure of these large corrections is simple enough that they can be summed to all orders, which we do in Section 5. The upshot is that the large corrections have the effect of renormalizing the ionization energy of the 
polymer, and once this is taken into account the remaining corrections are small, of order $15 \%$. Finally we gather some conclusions in Section 6 .

\section{Chain Dynamics in Formalism of Second Quantization}

The model of string bits proposed in Ref.[3,9] leads to the relativistic string in light-cone gauge. ${ }^{[1]}$ Although the string formed from string bits moves in $D$ space-time dimensions (described by light-cone coordinates

$$
\begin{aligned}
x^{ \pm} & =\left(x^{0} \pm x^{1}\right) / \sqrt{2} \\
\mathbf{x} & =\left(x^{2}, \cdots, x^{d}\right)
\end{aligned}
$$

where $d=D-1$ is the spatial dimension), the string bits move only in the transverse space, with coordinates $\mathbf{x}$ : in fact they enjoy a Newtonian (i.e. Galilean invariant) dynamics in this transverse space. The $x^{+} \equiv \tau$ coordinate is a Newtonian time for the string bits, but the longitudinal dimension, corresponding to $x^{-}$and its conjugate momentum $\mathrm{P}^{+}$, is nonexistent for them. It only emerges with string formation: bit number is a conserved quantity in string bit dynamics, and for a string with a large number of bits it becomes an effectively continuous variable, which can be consistently interpreted as a variable $P^{+}$. In this interpretation, we can think of each string bit as carrying a tiny fixed amount of $P^{+}=\epsilon$. Then a string containing $M$ string bits would carry $P^{+}=M \epsilon$. The fact that the $P^{-}$ of the string should vary as $1 / P^{+}$, is then understood as the $1 / M$ dependence of low energy excitations of large systems. The hamiltonian describing string bit dynamics will accordingly be identified as the operator $\epsilon P^{-}$for strings with an infinite number of string bits.

The string bit dynamics proposed in Ref.[3,9] exploits 't Hooft's ideas on the $1 / N$ expansion. ${ }^{[12]}$ We postulated that the string bit annihilation operator is an $N \times N$ matrix variable $a_{k}^{\ell}(\mathbf{x})$ and denoted the string bit creation operator by 
$\bar{a}_{k}^{\ell}(\mathbf{x})=a_{\ell}^{k}(\mathbf{x})^{\dagger}$. Then

$$
\left[a_{k}^{\ell}(\mathbf{x}), \bar{a}_{m}^{n}(\mathbf{y})\right]=\delta_{k}^{n} \delta_{m}^{\ell} \delta(\mathbf{x}-\mathbf{y})
$$

In the large $N$ limit, one can show that the singlet operators

$$
\bar{A}\left(\mathbf{x}_{1}, \cdots, \mathbf{x}_{M}\right)=\left(\frac{1}{N}\right)^{M / 2} \operatorname{tr}\left\{\bar{a}\left(\mathbf{x}_{1}\right) \cdots \bar{a}\left(\mathbf{x}_{M}\right)\right\}
$$

behave as creation operators for closed polymers of string bits. ${ }^{[13]}$ Note here that Bose statistics for the string bits implies cyclic symmetry for the $\bar{A}$ 's. This symmetry accounts for the well-known $L_{0}=\bar{L}_{0}$ constraint in the eventual low energy string dynamics.

A candidate dynamics for bosonic string bits is given by the Hamiltonian

$$
\begin{aligned}
H \equiv & \epsilon P^{-}=\int d \mathbf{x} \frac{1}{2} \operatorname{tr} \nabla \bar{a}(\mathbf{x}) \cdot \nabla a(\mathbf{x}) \\
& +\frac{1}{2 N} \int d \mathbf{x} d \mathbf{y} \mathcal{V}(\mathbf{x}-\mathbf{y}) \operatorname{tr}:(\bar{a}(\mathbf{x}) a(\mathbf{x})-a(\mathbf{x}) \bar{a}(\mathbf{x}))(\bar{a}(\mathbf{y}) a(\mathbf{y})-a(\mathbf{y}) \bar{a}(\mathbf{y})):
\end{aligned}
$$

where $\mathcal{V}$ should be positive (repulsive) for stability. Evaluating the action of $H$ on a state

$$
|\psi\rangle=\left(\frac{1}{N}\right)^{M / 2} \operatorname{tr}\left\{\bar{a}\left(\mathbf{x}_{1}\right) \cdots \bar{a}\left(\mathbf{x}_{M}\right)\right\} \psi_{M}\left(\mathbf{x}_{1}, \cdots, \mathbf{x}_{M}\right)|0\rangle
$$

leads to

$$
\begin{aligned}
H|\psi\rangle & =\left|\sum_{i}^{M}\left(-\nabla_{\mathbf{i}}^{2} / 2-\mathcal{V}\left(\mathbf{x}_{i+1}-\mathbf{x}_{i}\right)\right) \psi\right\rangle \\
- & \frac{1}{N} \sum_{i=1}^{M} \sum_{j \neq i, i+1} \bar{A}\left(\mathbf{x}_{i+1}, \cdots, \mathbf{x}_{j-1}\right) \bar{A}\left(\mathbf{x}_{j}, \cdots, \mathbf{x}_{i}\right)|0\rangle \mathcal{V}\left(\mathbf{x}_{i}-\mathbf{x}_{j}\right) \psi_{M}\left(\mathbf{x}_{1}, \cdots, \mathbf{x}_{M}\right) \\
& + \text { Other Terms of Order }(1 / \mathrm{N}) .
\end{aligned}
$$

The displayed $1 / N$ term describes the dissociation of a closed polymer into two closed polymers, so $1 / N$ can be identified with the string coupling constant. In 
the limit that it vanishes $(N \rightarrow \infty)$, the above equation describes a stable polymer provided that the nearest neighbor potential $-\mathcal{V}$ is sufficiently attractive to bind a pair of string bits. ${ }^{[3]}$ We shall review the argument below.

Our aim in this article is to go as far as we can toward the solution of this large $N$ limit of the string bit system. Referring to (2.2) we see that the energy eigenstates in this limit are determined by the solution of the following nonrelativistic many body Schrödinger equation:

$$
\sum_{i=1}^{M}\left(-\nabla_{\mathbf{i}}^{2} / 2-\mathcal{V}\left(\mathbf{x}_{i+1}-\mathbf{x}_{i}\right)\right) \psi\left(\mathbf{x}_{\mathbf{1}}, \ldots, \mathbf{x}_{\mathbf{M}}\right)=E \psi\left(\mathbf{x}_{\mathbf{1}}, \ldots, \mathbf{x}_{\mathbf{M}}\right)
$$

For purposes of keeping this article reasonably self-contained, the rest of this section will be devoted to a recapitulation of the main results already reported in Ref.[3]. New developments will be reserved for the subsequent sections.

It is first convenient to use Galilean invariance to separate the motion of the center of mass. So define

$$
\begin{aligned}
\mathbf{X} & \equiv \frac{1}{M} \sum_{r} \mathbf{x}_{r} \\
\mathbf{y}_{r} & \equiv \mathbf{x}_{r}-\mathbf{x}_{r-1}
\end{aligned}
$$

where $\mathbf{x}_{0} \equiv \mathbf{x}_{M}$ and $\mathbf{x}_{M+1} \equiv \mathbf{x}_{1}$. Note that although we have defined a $\mathbf{y}_{r}$ for each $r$, only $M-1$ of them are independent: they satisfy the constraint

$$
\mathbf{Y} \equiv \frac{1}{M} \sum_{r=1}^{M} \mathbf{y}_{r}=0
$$

Choosing $\mathbf{y}_{2}, \ldots, \mathbf{y}_{M}$ as independent variables, and denoting $(1 / i) \partial / \partial \mathbf{X} \equiv \mathbf{P}$ and $(1 / i) \partial / \partial \mathbf{y}_{r} \equiv \mathbf{p}_{r}$, the many body Hamiltonian corresponding to (2.3) becomes

$$
\begin{aligned}
h & =\frac{\mathbf{P}^{2}}{2 M}+\sum_{r=2}^{M} \mathbf{p}_{r}^{2}-\sum_{r=2}^{M-1} \mathbf{p}_{r} \cdot \mathbf{p}_{r+1}-\sum_{r=2}^{M} \mathcal{V}\left(\mathbf{y}_{r}\right)-\mathcal{V}\left(-\mathbf{y}_{2}-\cdots-\mathbf{y}_{M}\right) \\
& \equiv \frac{\mathbf{P}^{2}}{2 M}+h^{\prime}
\end{aligned}
$$

The first term on the r.h.s., giving the energy of center of mass motion, is precisely 
what is needed for the consistent interpretation of $M \epsilon$ as $P^{+}$and $h / \epsilon$ as $P^{-}$. The remainder of the r.h.s. $h^{\prime}$ should have the interpretation (for $M \rightarrow \infty$ ) as $\mathcal{M}^{2} / 2 M$ where $\mathcal{M}$ is the rest mass operator for the string. Since there will inevitably be a bulk contribution to the eigenvalues of $h^{\prime}$ proportional to the number of string bits $M$, we must subtract this contribution before the $1 / M$ behavior is revealed. This subtraction is easily accomplished by including a counterterm in the original string bit Hamiltonian of the form $\mu_{0} \int d \mathbf{x} \operatorname{tr} \bar{a}(\mathbf{x}) a(\mathbf{x})$. Note that in the low energy theory such a term is just a multiple of $P^{+}$. The remainder of this paper is devoted entirely to gaining an understanding of the eigenspectrum of $h^{\prime}$ especially the excitation energies of order $(1 / M)$ above the ground state energy.

We first use variational arguments to establish a simple criterion for the stability of the string bit polymers. Here we are assessing stability against the breaking of nearest neighbor bonds, i.e. the dissociation into a number of open polymers with all unbroken bonds retaining their arrangement. Dissociation of a closed polymer into two smaller closed polymers by bond rearrangement is another matter intimately associated with the presence or absence of tachyons in the low energy string theory. Such decays are vetoed by the $N \rightarrow \infty$ limit corresponding to zero string coupling constant.

We assume that the potential $-\mathcal{V}$ is negative and vanishes at large distances. The zero of energy corresponds to all the string bits infinitely dispersed from one another. Notice that deleting the last term in (2.5) leaves the Hamiltonian for an open polymer with the same number of bits. Since the deleted term is negative, this shows that the closed polymer ground state energy is strictly less than the open polymer ground state energy. Thus stability of the open polymer implies that of the closed polymer. Next consider the dissociation of an open polymer with $M$ bits into two open polymers with $M_{1}$ and $M-M_{1}$ bits respectively. Because of the nearest neighbor interaction structure, the internal Hamiltonian for an open polymer can be broken down as:

$h_{\text {Open }, M}^{\prime}=h_{\text {Open }, M_{1}}^{\prime}+h_{\text {Open }, M-M_{1}}^{\prime}+\mathbf{p}_{M_{1}+1}^{2}-\mathcal{V}\left(\mathbf{y}_{M_{1}+1}\right)-\mathbf{p}_{M_{1}} \cdot \mathbf{p}_{M_{1}+1}-\mathbf{p}_{M_{1}+1} \cdot \mathbf{p}_{M_{1}+2}$, 
where the variables labelled by $M_{1}+1$ do not appear in either of the open polymer Hamiltonians on the r.h.s. Thus applying the variational principle with a trial wave function given by $\psi_{\text {Trial }}=\psi_{M_{1}}^{G} \psi_{M-M_{1}}^{G} \psi_{2}^{G}\left(\mathbf{y}_{M_{1}+1}\right)$, where $\psi_{K}^{G}$ is the exact ground state wave function for a polymer with $K$ bits, gives

$$
E_{\text {Open }, M}^{G} \leq E_{\text {Open }, M_{1}}^{G}+E_{\text {Open }, M-M_{1}}^{G}+E_{2}^{G} .
$$

Here one uses the fact that $\left\langle\mathbf{p}_{r}\right\rangle=0$ in any of the ground state wave functions in the trial. If the nearest neighbor potential binds two bits, $E_{2}^{G}<0$ and the ionization energy required for dissociation into two open polymers is at least $B=-E_{2}^{G}$.

Now for large $M$, the ground state energy of an open polymer should have the behavior

$$
E_{\text {Open }, M}^{G} \sim \alpha M+\beta+\gamma / M+\cdots
$$

Inserting this form into the variational estimate gives the rigorous bound $\beta \geq$ $-E_{2}^{G}=B$. More precisely we see that $\beta$ has the interpretation as the ionization energy required to break a long open polymer into two smaller but still long open polymers. This ionization energy is just the energy cost of breaking a bond. Since the transformation of a closed polymer to an open polymer requires breaking precisely one bond, and for very long polymers the energy cost of this break can only differ from that of breaking an open polymer into two open polymers by energies of order $1 / M$, we can conclude that the ground state energy of a closed polymer should have the behavior

$$
E_{\text {Closed, } M}^{G} \sim \alpha M+\gamma^{\prime} / M
$$

The coefficients of the $1 / M$ terms are, of course, different in the open and closed cases. It is crucial for the interpretation of $h$ as $\epsilon P^{-}$that there is no constant $\beta$ term for the closed polymer ground state energy, since only the bulk $\alpha$ term can be removed by a counterterm in the string bit Hamiltonian. 
The important parameter that characterizes the stringy properties of long polymers is the string rest tension $T_{0}$. It can be inferred from the low lying level spacing of the polymers. Eigenvalues of $h^{\prime}$ of order $1 / M$ above the lowest eigenvalue will correspond to values of $P^{-}$behaving as $1 / P^{+}$. The coefficient of $1 / M$ is thus $2 \times \mathcal{M}^{2}$. To calculate $T_{0}$ it is simplest and sufficient to study long open polymers, and we shall make this restriction in what follows.

We only remark that the best way to apply the methods to the closed case is to consider a related system to $(2.5)$, in which one new degree of freedom $\left(\mathbf{y}_{1}, \mathbf{p}_{1}\right)$ is introduced, and the Hamiltonian is taken to be

$$
\hat{h}=\frac{\mathbf{P}^{2}}{2 M}+\sum_{r=1}^{M} \mathbf{p}_{r}^{2}-\sum_{r=1}^{M} \mathbf{p}_{r} \cdot \mathbf{p}_{r+1}-\sum_{r=1}^{M} \mathcal{V}\left(\mathbf{y}_{r}\right)
$$

To apply solutions of this system to (2.5), notice that the quantity $\mathbf{Y}$, defined below (2.4), commutes with $\hat{h}$. One can therefore look for eigenfunctions of $\hat{h}$ of the form $\delta(\mathbf{Y}) \psi\left(\mathbf{y}_{2}, \ldots, \mathbf{y}_{M}\right)$, whence $\psi$ must be an eigenfunction of (2.5). Thus the eigenstates of (2.5) are the subset of eigenstates of $\hat{h}$ which satisfy the constraint $\mathbf{Y}=0$.

Henceforth we restrict attention to the open polymer hamiltonian with center of mass motion removed

$$
h_{\text {Open }}^{\prime} \equiv \sum_{r=2}^{M} \mathbf{p}_{r}^{2}-\sum_{r=2}^{M-1} \mathbf{p}_{r} \cdot \mathbf{p}_{r+1}-\sum_{r=2}^{M} \mathcal{V}\left(\mathbf{y}_{r}\right)
$$

Our variational arguments have already established that the ground state of this system is a discrete bound state lying at least an amount $B$ below the continuum. We must next look for discrete levels lying $O(1 / M)$ above the ground level. For this purpose, we apply many body methods developed by Goldstone. ${ }^{[10,14]}$

Goldstone employs a second quantization formalism in which a fermionic field $\psi_{r}(\mathbf{y})$ is introduced for each bond $r=2, \ldots, M$ in the open polymer chain. Each bond number operator $M_{r}=\int d \mathbf{y} \psi_{r}^{\dagger}(\mathbf{y}) \psi_{r}(\mathbf{y})$ will be conserved by the second 
quantized dynamics and so can be consistently set to unity. In this sector the second quantized Hamiltonian

$$
\begin{aligned}
H_{\text {Open }}= & \int d \mathbf{y} \sum_{r=2}^{M} \psi_{r}^{\dagger}\left(-\nabla^{2}-\mathcal{V}(\mathbf{y})\right) \psi_{r} \\
& -\sum_{r=2}^{M-1} \int d \mathbf{y} \psi_{r}^{\dagger}(\mathbf{y})(-i \nabla) \psi_{r}(\mathbf{y}) \int d \mathbf{z} \cdot \psi_{r+1}^{\dagger}(\mathbf{z})(-i \nabla) \psi_{r+1}(\mathbf{z})
\end{aligned}
$$

is completely equivalent to (2.6). This Hamiltonian can now be analyzed in the interaction picture using Feynman diagrams in which the propagator is determined by the quadratic term and the vertex is determined by the quartic term. The conceptual advantage of this formalism is that the zeroth order ground state, namely the state in which each bond is in the ground state of its one body potential, is simply expressed as a boundary condition on the propagator, just as in the Dirac hole theory of positrons.

Expanding $\psi_{r}$ in eigenfunctions of the one body hamiltonian $\mathbf{p}^{2}-\mathcal{V}(\mathbf{y})$

$$
\psi_{r}(\mathbf{y})=\sum_{m>0} b_{m}^{r} \phi_{m}(\mathbf{y})+b_{0}^{r} \phi_{0}(\mathbf{y})
$$

where $m=0$ labels the ground state and $m>0$ labels the excited states, the zeroth order ground state is specified by

$$
b_{m}^{r}|0\rangle=0 \quad \text { for } m>0 \quad b_{0}^{r \dagger}|0\rangle=0 .
$$

Then the interaction picture propagator, represented by the first diagram in Fig. 1, is given by

$$
\begin{aligned}
\langle 0| T\left[\psi_{r}^{I}(\mathbf{y},\right. & \left.t) \psi_{s}^{I \dagger}(\mathbf{z}, 0)\right]|0\rangle \\
& =\delta_{r s}\left\{\theta(t) \sum_{m>0} \phi_{m}(\mathbf{y}) \phi_{m}^{*}(\mathbf{z}) e^{-i t E_{m}}-\theta(-t) \phi_{0}(\mathbf{y}) \phi_{0}^{*}(\mathbf{z}) e^{-i t E_{0}}\right\} \\
& =\delta_{r s} \int \frac{d \omega}{2 \pi} e^{-i \omega t}\left\{\sum_{m>0} \frac{-i \phi_{m}(\mathbf{y}) \phi_{m}^{*}(\mathbf{z})}{E_{m}-\omega-i \epsilon}+\frac{-i \phi_{0}(\mathbf{y}) \phi_{0}^{*}(\mathbf{z})}{E_{0}-\omega+i \epsilon}\right\} .
\end{aligned}
$$


FIG. 1. Feynman rules for a string bit chain.

The vertex function represented by the second diagram in Fig. 1 is given by

$$
i \delta_{r_{1} s_{1}} \delta_{r_{2} s_{2}}\left(\delta_{r_{1}, r_{2}+1}+\delta_{r_{1}, r_{2}-1}\right)\left(-i \nabla_{1}\right)\left(-i \nabla_{2}\right)
$$

where the gradient operators act on the first arguments of the respective propagators directed into the vertex, and a Kronecker delta with either index equal to 1 or $M+1$ is understood to vanish.

In order to apply time dependent perturbation theory to the calculation of ground state expectation values of time ordered products of operators like $\mathbf{y}_{r}$ or $\mathbf{p}_{r}$, we simply use Dyson's formula after translating to the second quantized formalism. Thus we have

$$
\begin{aligned}
\mathbf{y}_{r} & \equiv \int d \mathbf{y} \psi_{r}^{\dagger}(\mathbf{y}) \mathbf{y} \psi_{r}(\mathbf{y}) \\
\mathbf{p}_{r} & \equiv \int d \mathbf{y} \psi_{r}^{\dagger}(\mathbf{y}) \frac{1}{i} \frac{\partial}{\partial \mathbf{y}} \psi_{r}(\mathbf{y})
\end{aligned}
$$

so, for example,

$$
\begin{aligned}
& \left\langle G\left|T\left[y_{r}^{k}(t) y_{s}^{m}(0)\right]\right| G\right\rangle= \\
& \frac{\left\langle 0\left|T\left[\int d \mathbf{y} \psi_{r}^{I \dagger}(\mathbf{y}, t) y^{k} \psi_{r}^{I}(\mathbf{y}, t) \int d \mathbf{y} \psi_{s}^{I^{\dagger}}(\mathbf{z}, 0) z^{m} \psi_{r}^{I}(\mathbf{z}, 0) e^{-i \int_{-\infty}^{+\infty} d t^{\prime} H_{I}^{\prime}\left(t^{\prime}\right)}\right]\right| 0\right\rangle}{\left\langle 0\left|T\left[e^{-i \int_{-\infty}^{+\infty} d t^{\prime} H_{I}^{\prime}\left(t^{\prime}\right)}\right]\right| 0\right\rangle}
\end{aligned}
$$

where $H_{I}^{\prime}\left(t^{\prime}\right)$ is just the quartic term of (2.7) transformed to interaction picture. As usual the denominator in (2.10) simply cancels all disconnected bubble dia- 
grams and correctly normalizes the matrix element after the infinite time integral projects onto the true ground state of the many body system. Information on the spectrum of the exact Hamiltonian can be inferred from the time dependence of such correlation functions, or equivalently from the pole locations in their time Fourier transforms.

By applying the Random Phase Approximation corresponding to summing the bubble diagrams shown in Fig. 2, Goldstone demonstrated the existence of order $1 / M$ excitations in this approximation and gave an approximate formula for the string tension. In the following section we put these insights into an all orders context, and so obtain an exact formula for the string tension.

FIG. 2. Diagrams summed in the random phase approximation

\section{Low Energy Excitations: The String Tension}

Consider the system

$$
h_{\text {Open }}^{\prime}=\sum_{r=2}^{M}\left[\mathbf{p}_{r}^{2}+\mathcal{V}\left(\mathbf{y}_{r}\right)\right]-\sum_{r=2}^{M-1} \mathbf{p}_{r} \cdot \mathbf{p}_{r+1}
$$

Our aim is to calculate the low lying excitations of such a system under the assumption that the system binds together, i.e. that there is a discrete ground state with a mass gap. We shall examine the poles in the time Fourier transforms of 
various correlation functions:

$$
\begin{aligned}
\left\langle G\left|T\left[y_{r}^{i}(t) y_{s}^{j}(0)\right]\right| G\right\rangle & \equiv-\int \frac{d \omega}{2 \pi i} \mathcal{E}_{r s}^{i j}(\omega) e^{-i \omega t} \\
\left\langle G\left|T\left[p_{r}^{i}(t) p_{s}^{j}(0)\right]\right| G\right\rangle & \equiv-\int \frac{d \omega}{2 \pi i} \mathcal{G}_{r s}^{i j}(\omega) e^{-i \omega t} \\
\left\langle G\left|T\left[y_{r}^{i}(t) p_{s}^{j}(0)\right]\right| G\right\rangle & \equiv-\int \frac{d \omega}{2 \pi i} \mathcal{F}_{r s}^{y p, i j}(\omega) e^{-i \omega t} \\
\left\langle G\left|T\left[p_{r}^{i}(t) y_{s}^{j}(0)\right]\right| G\right\rangle & \equiv-\int \frac{d \omega}{2 \pi i} \mathcal{F}_{r s}^{p y, i j}(\omega) e^{-i \omega t}
\end{aligned}
$$

Using time translation invariance, we can transfer the time argument to the other member of the time ordered product, which leads to the identities

$$
\begin{gathered}
\mathcal{G}_{r s}(-\omega)=\mathcal{G}_{s r}(\omega) \\
\mathcal{E}_{r s}(-\omega)=\mathcal{E}_{s r}(\omega) \\
\mathcal{F}_{r s}^{p y}(-\omega)=\mathcal{F}_{s r}^{y p}(\omega) .
\end{gathered}
$$

Using the Heisenberg equations of motion we easily derive the "Ward identities":

$$
\begin{aligned}
\frac{d}{d t}\left\langle G\left|T\left[y_{r}^{i}(t) y_{s}^{j}(0)\right]\right| G\right\rangle & =\left\langle G\left|T\left[\left(2 p_{r}^{i}(t)-p_{r+1}^{i}(t)-p_{r-1}^{i}(t)\right) y_{s}^{j}(0)\right]\right| G\right\rangle \\
\frac{d}{d t}\left\langle G\left|T\left[y_{r}^{i}(t) p_{s}^{j}(0)\right]\right| G\right\rangle & =i \delta_{i j} \delta_{r s} \delta(t)+\left\langle G\left|T\left[\left(2 p_{r}^{i}(t)-p_{r+1}^{i}(t)-p_{r-1}^{i}(t)\right) p_{s}(0)\right]\right| G\right\rangle
\end{aligned}
$$

Taking Fourier transforms of the Ward identities, defining $k_{r s}^{2}=2 \delta_{r s}-\delta_{r s+1}-\delta_{r s-1}$, leads to

$$
\begin{gathered}
-i \omega \mathcal{E}^{i j}(\omega)=k^{2} \mathcal{F}_{p y}^{i j}(\omega) \\
-i \omega \mathcal{F}_{y p}^{i j}(\omega)=\delta_{i j} I+k^{2} \mathcal{G}^{i j}(\omega) \\
i \omega \mathcal{F}_{p y}^{i j}(\omega)=\delta_{i j} I+\mathcal{G}^{i j}(\omega) k^{2} \\
i \omega \mathcal{E}^{i j}(\omega)=\mathcal{F}_{y p}^{i j}(\omega) k^{2}
\end{gathered}
$$

The bond labels $r, s$ have been suppressed in writing these equations, i.e. each of the quantities $\mathcal{E}, \mathcal{F}_{p y}$, and $\mathcal{G}$ is an $(M-1) \times(M-1)$ matrix, and the multiplications 
indicated in these equations are matrix multiplications. Corresponding to $\mathcal{E}, \mathcal{F}_{p y}$, and $\mathcal{G}$, we shall introduce "irreducible parts" $\Pi_{y y}, \Pi_{p y}, \Pi_{y p}, \Pi_{p p}$. Referring to the Feynman diagram expansion of Section 2, these quantities are defined as the sums of all diagrams, contributing to the respective correlation function, which cannot be cut in two by cutting a single dashed line. See Fig. 1. Then we can write an equation for each of the quantities $\mathcal{E}, \mathcal{F}_{p y}$, and $\mathcal{G}$ as follows:

$$
\begin{gathered}
\mathcal{G}^{i k}(\omega)=\Pi_{p p}^{i j}(\omega)\left[\delta_{j k}+\left(k^{2}-2\right) \mathcal{G}^{j k}\right] \\
\mathcal{F}_{y p}^{i k}(\omega)=\Pi_{y p}^{i j}(\omega)\left[\delta_{j k}+\left(k^{2}-2\right) \mathcal{G}^{j k}\right] \\
\mathcal{F}_{p y}^{i k}(\omega)=\left[\delta_{i j}+\mathcal{G}^{i j}\left(k^{2}-2\right)\right] \Pi_{p y}^{j k} \\
\mathcal{E}^{i k}(\omega)=\Pi_{y y}^{i k}+\mathcal{F}_{y p}^{i j}\left(k^{2}-2\right) \Pi_{p y}^{j k}
\end{gathered}
$$

This set of equations gives a purely algebraic determination of the irreducible parts in terms of the full correlation functions.

Next we wish to use the Ward Identities to derive relationships among the various $\Pi$ 's. First use $(3.2)$ and $(3.6)$ to eliminate $\mathcal{F}_{y p}$ :

$$
\delta_{i j} I+k^{2} \mathcal{G}^{i j}(\omega)=-i \omega \Pi_{y p}^{i k}(\omega)\left[\delta_{k j}+\left(k^{2}-2\right) \mathcal{G}^{k j}\right]
$$

and then (3.5) can be used to rewrite the l.h.s. of (3.9):

$$
\begin{aligned}
\delta_{i j} I+k^{2} \mathcal{G}^{i j}(\omega) & =\delta_{i j} I+\left(k^{2}-2\right) \mathcal{G}^{i j}+2 \Pi_{p p}^{i k}\left[\delta_{k j}+\left(k^{2}-2\right) \mathcal{G}^{k j}\right] \\
& =\left[\delta_{i k}+2 \Pi_{p p}^{i k}\right]\left(\delta_{k j}+\left(k^{2}-2\right) \mathcal{G}^{k j}\right) .
\end{aligned}
$$

Putting this into (3.9) gives,

$$
\left[\delta_{i k}+2 \Pi_{p p}^{i k}+i \omega \Pi_{y p}^{i k}\right]\left(\delta_{k j}+\left(k^{2}-2\right) \mathcal{G}^{k j}\right)=0
$$

Now provided $\delta_{k j}+\left(k^{2}-2\right) \mathcal{G}^{k j}$ is invertible, we obtain our first relation:

$$
-i \omega \Pi_{y p}^{i j}=\delta_{i j}+2 \Pi_{p p}^{i j}
$$

Next, the identities $\mathcal{G}(-\omega)=\mathcal{G}^{T}(\omega)$ and $\mathcal{F}_{p y}(\omega)=\mathcal{F}_{y p}^{T}(-\omega)$ yield the transposed 
relationship:

$$
i \omega \Pi_{p y}^{i j}(\omega)=\delta_{i j} I+2 \Pi_{p p}^{i j}(\omega)
$$

The last relation we seek is between $\Pi_{y y}$ and $\Pi_{p p}$. First insert (3.4) in (3.8) to obtain

$$
\mathcal{E}^{i k}(\omega)=\Pi_{y y}^{i k}+i \omega \mathcal{E}^{i j}(\omega) \frac{k^{2}-2}{k^{2}} \Pi_{p y}^{j k}(\omega)
$$

Then use (3.11) to elimintate $\Pi_{p y}$,

$$
\mathcal{E}^{i k}(\omega)=\Pi_{y y}^{i k}+\mathcal{E}^{i j}(\omega) \frac{k^{2}-2}{k^{2}}\left(\delta_{j k} I+2 \Pi_{p p}^{j k}(\omega)\right)
$$

Leading to

$$
\Pi_{y y}^{i k}(\omega)=\mathcal{E}^{i j}(\omega)\left[\frac{2}{k^{2}} \delta_{j k} I-\frac{k^{2}-2}{k^{2}} 2 \Pi_{p p}^{j k}(\omega)\right] .
$$

(3.2) and (3.4) together give

$$
\omega^{2} \mathcal{E}^{i j} \frac{1}{k^{2}}=\delta_{i j} I+k^{2} \mathcal{G}^{i j}(\omega)
$$

which, inserted into (3.12), yields

$$
\begin{aligned}
\omega^{2} \Pi_{y y}^{i k}(\omega) & =2\left[\delta_{i j} I+k^{2} \mathcal{G}^{i j}(\omega)\right]\left[\delta_{j k} I-\left(k^{2}-2\right) \Pi_{p p}^{j k}(\omega)\right] \\
& =2 \delta_{i k} I+2 k^{2} \mathcal{G}^{i k}(\omega)-2\left(k^{2}-2\right) \Pi_{p p}^{i k}(\omega)-2 k^{2} \mathcal{G}^{i j}(\omega)\left(k^{2}-2\right) \Pi_{p p}^{j k}
\end{aligned}
$$

Now putting $(\omega \rightarrow-\omega)$ and taking the transpose of (3.5) shows that

$$
\mathcal{G}^{i k}-\Pi_{p p}^{i k}=\mathcal{G}^{i j}\left(k^{2}-2\right) \Pi_{p p}^{j k}
$$

Using this result in the last term of (3.13) gives, after some simplification, the last 
desired relationship:

$$
\omega^{2} \Pi_{y y}^{i k}(\omega)=2 \delta_{i k} I+4 \Pi_{p p}^{i k}(\omega)
$$

or, rearranging a bit,

$$
\Pi_{p p}^{i k}(\omega)=-\frac{1}{2} \delta_{i k} I+\frac{\omega^{2}}{4} \Pi_{y y}^{i k}(\omega) .
$$

The implications of the Ward identities on the irreducible parts are summarized in (3.10), (3.11), (3.14).

Armed with these results, we now turn to their implications for the low lying excitations of the polymer system. This information is given by the location of poles in $\mathcal{G}$, or by the zero eigenvalues of its inverse, which, for $\omega \approx 0$, is proportional to

$$
\begin{aligned}
\delta_{j k}-\Pi_{j k}^{p p}(\omega)\left(k^{2}-2\right) & =\delta_{j k}+\frac{1}{2}\left(k^{2}-2\right) \delta_{j k}-\frac{\omega^{2}}{4} \Pi_{y y}^{j k}\left(k^{2}-2\right) \\
& =\frac{1}{2} k^{2} \delta_{j k}-\frac{\omega^{2}}{4} \Pi_{y y}^{j k}(\omega)\left(k^{2}-2\right) .
\end{aligned}
$$

The matrix $k^{2}$ has eigenvalues of $O\left(1 / M^{2}\right)$ as $M \rightarrow \infty$, so there are eigenfrequencies $\omega^{2}$ of the same order. For these we may neglect $k^{2}$ in the second term of (3.15) and evaluate $\Pi_{y y}$ at $\omega=0$. We therefore must search for zero eigenvalues of the matrix

$$
\omega^{2} \Pi_{y y}^{i k}(0)+k^{2} \delta_{i k}
$$

as $M \rightarrow \infty$. It is interesting and useful to note that by virtue of the Ward identities, $\mathcal{E}^{i k}(0)=\Pi_{y y}^{i k}(0)$. Thus there is no a priori requirement to separate out the irreducible part: at zero frequency the reducible parts vanish identically. Finally, by rotational invariance, we can write

$$
\Pi_{r s}^{i k}(\omega)=\delta_{i k} \Pi_{r s}(\omega)
$$

so that (3.17) can be simplified to

$$
\omega^{2} \Pi_{y y}(0)+k^{2}
$$


To examine the large $M$ dependence of (3.15) go to normal modes:

$$
\begin{aligned}
\Pi_{m n} & =\frac{2}{M} \sum_{r, s} \sin \frac{m \pi r}{M} \Pi_{r s} \sin \frac{n \pi s}{M} \\
k_{m n}^{2} & =\delta_{m n} 2\left(1-\cos \frac{n \pi}{M}\right),
\end{aligned}
$$

after which the r.h.s. of (3.15) becomes

$$
\delta_{j k} \delta_{m n}\left(1-\cos \frac{n \pi}{M}\right)+\frac{\omega^{2}}{2} \Pi_{m n}(\omega) \delta_{j k} \cos \frac{n \pi}{M}
$$

To get the spectrum of the relativistic string as $M \rightarrow \infty$, we should find that $\omega \propto n / M$, which requires that $\Pi_{m n}(0)$ must be diagonal and independent of $m$ for small $m / M$ or $n / M$. The condition for this to be true is that, away from $r=2, M$, $\Pi_{r s} \rightarrow 0$ sufficiently rapidly as $|r-s| \rightarrow \infty$.

To see this, calculate

$$
\Pi_{m n}=\frac{2}{M} \sum_{r, s} \Pi_{r+s, s} \sin \frac{m \pi(r+s)}{M} \sin \frac{n \pi s}{M} .
$$

For $r, s$ away from the endpoints, $\Pi_{r s}$ should be only a function of $r-s$, so denoting it as $\Pi(r-s)$, we have

$$
\begin{aligned}
\Pi_{m n} & =\frac{2}{M} \sum_{s, r} \Pi(r) \sin \frac{n \pi s}{M}\left(\sin \frac{m \pi s}{M} \cos \frac{m \pi r}{M}+\cos \frac{m \pi s}{M} \sin \frac{m \pi r}{M}\right) \\
& =\delta_{m n}\left(\sum_{r} \cos \frac{m \pi r}{M} \Pi(r)\right)+\left(\sum_{r} \Pi(r) \sin \frac{m \pi r}{M}\right) \frac{2}{M} \sum_{s} \sin \frac{n \pi s}{M} \cos \frac{m \pi s}{M} \\
& \approx \delta_{m n}\left[\sum_{r} \Pi(r)\right]
\end{aligned}
$$

as $M \rightarrow \infty$, provided $\Pi^{j k}(r)$ vanishes rapidly enough as $r \rightarrow \infty$. This is certainly true to any finite order of perturbation theory. Making this assumption 
nonperturbatively, we have,

$$
\omega^{2} \sum_{r} \Pi(r) \approx-2\left(1-\cos \frac{m \pi}{M}\right)=-4 \sin ^{2} \frac{m \pi}{2 M} \approx-\frac{m^{2} \pi^{2}}{(M)^{2}} .
$$

Since $\omega$ is to be identified with $\epsilon P^{-}$, and $P^{+}=\epsilon M$, the $(\text { mass })^{2}=2 P^{+} P^{-}$is given by $2 m \pi / \sqrt{-\sum_{r} \Pi(r)}$, by definition, the coefficient of $m$ in this formula is $1 / \alpha^{\prime}=2 \pi T_{0}$ :

$$
T_{0}=\frac{B}{\sqrt{-\sum_{r} \Pi(r)}} \quad \alpha^{\prime}=\frac{1}{2 \pi B} \sqrt{-\sum_{r} \Pi(r)}
$$

\section{Random Phase Approximation and First Corrections}

The Random Phase Approximation (RPA) is to calculate $\Pi_{r s}^{i j}$ replacing $H$ by $H_{0}$ :

$$
\Pi_{\mathrm{RPA}, r s}^{i j}(0)=\delta_{r s} \Pi^{i j}(0)
$$

where

$$
\Pi^{i j}(0)=-i \int_{-\infty}^{\infty} d t\left\langle 0\left|T\left[y^{i}(t) y^{j}(0)\right]\right| 0\right\rangle
$$

corresponding to the one body problem

$$
H_{1 \text { body }}=\mathbf{p}^{2}-\mathcal{V}(\mathbf{y})
$$

Putting in a complete set of states:

$$
\begin{aligned}
\Pi^{i j}(0)=-i \int_{-\infty}^{\infty} d t\left[\theta(t) \sum_{n \neq 0}\left\langle 0\left|y^{i}(0)\right| n\right\rangle\left\langle n\left|y^{j}(0)\right| 0\right\rangle e^{-i\left(E_{n}-E_{0}\right) t}+\right. \\
\theta(-t) \sum_{n \neq 0}\left\langle 0\left|y^{j}(0)\right| n\right\rangle\left\langle n\left|y^{i}(0)\right| 0\right\rangle e^{+i\left(E_{n}-E_{0}\right) t} \\
=-2 \sum_{n \neq 0} \frac{\langle 0|\mathbf{y}| n\rangle \cdot\langle n|\mathbf{y}| 0\rangle}{E_{n}-E_{0}}\left(\frac{\delta_{i j}}{2}\right)
\end{aligned}
$$




$$
\omega_{m}^{\mathrm{RPA}}\left\{\sum_{n \neq 0} \frac{\mathbf{y}_{0 n} \cdot \mathbf{y}_{n 0}}{E_{n}-E_{0}}\right\}^{1 / 2} \approx \frac{m \pi}{M}
$$

From which, the Regge slope is

$$
\alpha_{\mathrm{RPA}}^{\prime}=\frac{1}{2 \pi}\left\{\sum_{m \neq 0} \frac{\mathbf{y}_{0 n} \cdot \mathbf{y}_{n 0}}{E_{n}-E_{0}}\right\}^{1 / 2}
$$

a result first obtained by Goldstone. ${ }^{[14]}$

On the face of it, it would seem that the validity of RPA depends on the smallness of the perturbation. That this is not necessarily so is shown by the fact that for $-\mathcal{V}=K \mathbf{y}^{2}$ the RPA gives the exact result for the string tension, even though the ground state energy receives finite corrections. This is by no means transparent in terms of diagrams: the corrections to the string tension cancel, apparently miraculously. One can expect that potentials that are close to quadratic near the minimum will lead to a quite accurate RPA.

However, probably the most interesting candidate string bit potential is a local one, $\mathcal{V}(\mathbf{y})=\lambda_{0} \delta(\mathbf{y})$. In two transverse dimensions (corresponding to four dimensional space-time!), this is a näively scale invariant potential, although ultraviolet divergences give logarithmic scaling violations. Scale invariance is a desirable property of the microscopic potential since, as Gross and Mende ${ }^{[15]}$ and others have emphasized, it should be enjoyed by string theory at short distances. Even apart from its appeal for string bit dynamics, this potential is drastically different from a quadratic one, which makes it a good test case for assessing the accuracy of RPA. Thus in this section we consider corrections to RPA for this special potential.

We begin by reviewing the basic features of the one body problem represented by

$$
h_{1 \text { body }}=\mathbf{p}^{2}-\lambda_{0} \delta(\mathbf{y})
$$

as it determines the zeroth order in time dependent perturbation theory. First consider the bound state problem. For an energy eigenvalue $E=-B, B>0$, the 
momentum space wave function is easily shown to be given by

$$
\phi(\mathbf{p})=\frac{\lambda_{0} \psi(\mathbf{0})}{\mathbf{p}^{2}+B}
$$

Since $\psi(\mathbf{0})=\int d \mathbf{p} \phi(\mathbf{p}) /(2 \pi)^{2}$, the quantization condition for $B$ is obtained by integrating both sides over $\mathbf{p}$ :

$$
1=\lambda_{0} \int \frac{d \mathbf{p}}{4 \pi^{2}} \frac{1}{\mathbf{p}^{2}+B}
$$

In two dimensions the integral diverges in the ultraviolet, which requires asymptotic freedom $\left(\lambda_{0} \rightarrow 0\right)$ in order for $B$ to be finite. With an ultraviolet cutoff $\Lambda$ on the momentum integral, we find

$$
B=\Lambda^{2} e^{-4 \pi / \lambda_{0}}
$$

that is we have dimensional transmutation, ${ }^{[3]}$ in which the dimensionless $\lambda_{0}$ is traded for the dimensionful $B$. In the limit $\Lambda \rightarrow \infty, \psi(\mathbf{0})$ is infinite but $\lambda_{0} \psi(\mathbf{0})$ is finite. The normalized wave function is

$$
\begin{aligned}
\psi(\mathbf{y}) \equiv R_{B}(|\mathbf{y}|) & =\sqrt{\frac{B}{\pi}} \int \frac{d \mathbf{p}}{2 \pi} \frac{e^{i \mathbf{p} \cdot \mathbf{y}}}{\mathbf{p}^{2}+B} \\
& =\sqrt{\frac{B}{\pi}} K_{0}(|\mathbf{y}| \sqrt{B}),
\end{aligned}
$$

where $K_{0}$ is the zero order modified Bessel function of the third kind.

Clearly this zero angular momentum state is the only discrete state. The rest of the $s$-wave spectrum is positive energy continuum. For each energy $E \equiv k^{2}$, there is one state whose wave function is that unique linear combination of Bessel functions $J_{0}(k|\mathbf{y}|)$ and $Y_{0}(k|\mathbf{y}|)=N_{0}(k|\mathbf{y}|)$ which is orthogonal to (4.3):

$$
R_{k}(|\mathbf{y}|)=\frac{1}{\sqrt{1+\pi^{2} / \ln ^{2}\left(B / k^{2}\right)}}\left[J_{0}(k|\mathbf{y}|)+\frac{\pi}{\ln \left(B / k^{2}\right)} Y_{0}(k|\mathbf{y}|)\right]
$$

where we have normalized $R_{k}$ according to

$$
\int d \mathbf{y} R_{k}(|\mathbf{y}|) R_{k^{\prime}}(|\mathbf{y}|)=\frac{2 \pi}{k} \delta\left(k-k^{\prime}\right)
$$

The radial wave functions of the states with nonzero angular momentum $L_{z}=m$ 
vanish at the origin and so coincide with the corresponding free wave functions, namely $R_{k}^{m}(|\mathbf{y}|)=J_{m}(k|\mathbf{y}|)$.

From the above information we can easily construct the Green function

$$
G_{E}(\mathbf{y}, \mathbf{z}) \equiv\left\langle\mathbf{y}\left|\frac{1}{h_{1 \text { body }}-E-i \epsilon}\right| \mathbf{z}\right\rangle
$$

The $s$-wave contribution to $G_{E}$ is just

$$
G_{E}^{s-\text { wave }}(\mathbf{y}, \mathbf{z})=\frac{R_{B}(|\mathbf{y}|) R_{B}(|\mathbf{z}|)}{-B-E-i \epsilon}+\int_{0}^{\infty} \frac{k d k}{2 \pi} \frac{R_{k}(|\mathbf{y}|) R_{k}(|\mathbf{z}|)}{k^{2}-E-i \epsilon} .
$$

The non $s$-wave contribution is just the free Green function minus its $s$-wave. Thus we have

$$
\begin{aligned}
G_{E}(\mathbf{y}, \mathbf{z})=\int \frac{d \mathbf{p}}{(2 \pi)^{2}} \frac{e^{i \mathbf{p} \cdot(\mathbf{y}-\mathbf{z})}}{\mathbf{p}^{2}-E-i \epsilon}+\frac{R_{B}(|\mathbf{y}|) R_{B}(|\mathbf{z}|)}{-B-E-i \epsilon} \\
+\int_{0}^{\infty} \frac{k d k}{2 \pi} \frac{R_{k}(|\mathbf{y}|) R_{k}(|\mathbf{z}|)-J_{0}(k|\mathbf{y}|) J_{0}(k|\mathbf{z}|)}{k^{2}-E-i \epsilon}
\end{aligned}
$$

By working directly with the two dimensional partial differential equation that determines $G_{E}$, it is straightforward to show that an alternate representation is

$$
\begin{aligned}
G_{E}(\mathbf{y}, \mathbf{z})=\int \frac{d \mathbf{p}}{(2 \pi)^{2}} \frac{e^{i \mathbf{p} \cdot(\mathbf{y}-\mathbf{z})}}{\mathbf{p}^{2}-E-i \epsilon} \\
\quad+\frac{1}{\pi \ln (-(E+i \epsilon) / B)} K_{0}(|\mathbf{y}| \sqrt{-E-i \epsilon}) K_{0}(|\mathbf{z}| \sqrt{-E-i \epsilon})
\end{aligned}
$$

Apart from the distribution of $i \epsilon$ 's, $G_{\omega}$ is just the Fourier transform of the propagator (2.8) needed for developing time dependent perturbation theory. To get the right boundary conditions for the propagator, one simply has to change the $-i \epsilon$ in the middle term of (4.6) to a $+i \epsilon$. To keep track of this change, it is convenient to 
define a reduced Green function $\tilde{G}_{E}$ which doesn't have the bound state pole pole:

$$
\tilde{G}_{E} \equiv G_{E}-\frac{R_{B}(|\mathbf{y}|) R_{B}(|\mathbf{z}|)}{-B-E-i \epsilon}
$$

Then the propagator (2.8) can be expressed as

$$
\begin{aligned}
\left\langle 0\left|T\left[\psi_{r}^{I}(\mathbf{y}, t) \psi_{s}^{I \dagger}(\mathbf{z}, 0)\right]\right| 0\right\rangle & =-i \delta_{r s} \int \frac{d \omega}{2 \pi} e^{-i \omega t}\left[\tilde{G}_{\omega}+\frac{R_{B}(|\mathbf{y}|) R_{B}(|\mathbf{z}|)}{-B-\omega+i \epsilon}\right] \\
& \equiv-i \delta_{r s} \int \frac{d \omega}{2 \pi} e^{-i \omega t} \hat{G}_{\omega}
\end{aligned}
$$

With this explicit representation for the propagator we are now in a position to write down and evaluate the expressions assigned to the first few diagrams in time dependent perturbation theory.

Focusing on the string tension, we have seen in the previous section that we need to compute $\Pi_{y y}$ at zero frequency. To lowest order in perturbation theory, we must evaluate only the first diagram of Fig. 2. (The remaining diagrams in Fig. 2 are reducible, and in any case vanish at zero frequency.) In next order we have the diagrams of Fig. 3. To this order irreducible diagrams with the external sources attached to different lines vanish by rotational invariance.

FIG. 3. First order corrections to RPA. 
Turning first to the lowest order diagram, the Feynman rules assign to this diagram the expression

$$
\begin{aligned}
& (-i)(-i)^{2}(-1) \delta_{r s} \int \frac{d \omega}{2 \pi} \int d \mathbf{y} d \mathbf{z} \\
& \quad y^{j}\left(\tilde{G}_{\omega}(\mathbf{y}, \mathbf{z})+\frac{R_{B}(\mathbf{y}) R_{B}(\mathbf{z})}{-B-\omega+i \epsilon}\right) z^{k}\left(\tilde{G}_{\omega}(\mathbf{z}, \mathbf{y})+\frac{R_{B}(\mathbf{z}) R_{B}(\mathbf{y})}{-B-\omega+i \epsilon}\right) .
\end{aligned}
$$

The prefactor phases have the following origins: the first $-i$ from the definition of $\Pi$, the $(-i)^{2}$ from the two propagators, and the $(-1)$ from the closed fermion loop. The $\omega$ integral can immediately be done by closing the contour in the upper half $\omega$ plane, picking up the pole at $\omega=-B+i \epsilon$. (The singularities from $\tilde{G}$ are all in the lower half plane.) The result is

$$
-2 \delta_{r s} \int d \mathbf{y} d \mathbf{z} R_{B}(\mathbf{y}) y^{j} \tilde{G}_{-B}(\mathbf{y}, \mathbf{z}) z^{k} R_{B}(\mathbf{z}),
$$

which is identical to the result of the previous section. Because $R_{B}$ is an $s$-wave state, the presence of $y^{j}$ and $z^{k}$ cause all $s$-wave contributions to $\tilde{G}$ to decouple, so the latter can be replaced with the free Green function. Working in momentum space we get

$$
-2 \delta_{r s} \frac{\delta_{j k}}{2} \frac{B}{\pi} \int d \mathbf{p} \frac{4 \mathbf{p}^{2}}{\left(\mathbf{p}^{2}+B\right)^{5}}=-4 B \delta_{r s} \delta_{j k} \int_{B}^{\infty} \frac{v-B}{v^{5}} d v=-\frac{1}{3 B^{2}} \delta_{r s} \delta_{j k} .
$$

This approximation for $\Pi_{y y}$ is, as we have discussed, the random phase approximation, giving a string tension of $T_{0} \equiv 1 / 2 \pi \alpha^{\prime} \approx T_{0}^{R P A}=B \sqrt{3}$.

Corrections to RPA are given by the three diagrams in Fig. 3. The first diagram represents the expression

$$
\begin{aligned}
& -2 \delta_{r s} i(-i)^{6}(-1)^{2} i^{2} \int d \mathbf{y} d \mathbf{z} d \mathbf{u} d \mathbf{v} d \mathbf{x} d \mathbf{w} \int \frac{d \omega_{1}}{2 \pi} \frac{d \omega_{2}}{2 \pi} \frac{d \omega_{3}}{2 \pi} \\
& y^{j} \hat{G}_{\omega_{2}}(\mathbf{y}, \mathbf{u})\left(-i \nabla_{u}^{m}\right) \hat{G}_{\omega_{1}}(\mathbf{u}, \mathbf{z}) z^{k} \hat{G}_{\omega_{1}}(\mathbf{z}, \mathbf{v})\left(-i \nabla_{v}^{n}\right) \hat{G}_{\omega_{2}}(\mathbf{v}, \mathbf{y}) \\
& \left(-i \nabla_{x}^{n}\right) \hat{G}_{\omega_{1}-\omega_{2}+\omega_{3}}(\mathbf{x}, \mathbf{w})\left(-i \nabla_{w}^{m}\right) \hat{G}_{\omega_{3}}(\mathbf{w}, \mathbf{x})
\end{aligned}
$$

The factor of two comes from the two possible terms in the vertex function (2.9); this assumes that $r, s$ are well away from 2 and $M$. To begin reducing this ex- 
pression first note that the $\omega_{3}$ integral can be closed in the upper half plane where there is only a simple pole at $\omega_{3}=-B+i \epsilon$. Thus

$$
\begin{aligned}
& \int \frac{d \omega_{3}}{2 \pi} \int d \mathbf{x} d \mathbf{w}\left(-i \nabla_{x}^{n}\right) \hat{G}_{\omega_{1}-\omega_{2}+\omega_{3}}(\mathbf{x}, \mathbf{w})\left(-i \nabla_{w}^{m}\right) \hat{G}_{\omega_{3}}(\mathbf{w}, \mathbf{x})= \\
& -i \int d \mathbf{x} d \mathbf{w}\left[R_{B}(\mathbf{x})\left(-i \nabla_{x}^{n}\right) \tilde{G}_{\omega_{1}-\omega_{2}-B}(\mathbf{x}, \mathbf{w})\left(-i \nabla_{w}^{m}\right) R_{B}(\mathbf{w})+\right. \\
& \left.R_{B}(\mathbf{w})\left(-i \nabla_{w}^{m}\right) \tilde{G}_{\omega_{2}-\omega_{1}-B}(\mathbf{w}, \mathbf{x})\left(-i \nabla_{x}^{n}\right) R_{B}(\mathbf{x})\right] \\
= & -i \frac{B}{\pi} \int d \mathbf{p} \frac{p^{m} p^{n}}{\left(\mathbf{p}^{2}+B\right)^{2}}\left[\frac{1}{\mathbf{p}^{2}+B-\omega_{1}+\omega_{2}-2 i \epsilon}+\frac{1}{\mathbf{p}^{2}+B-\omega_{2}+\omega_{1}-2 i \epsilon}\right] \\
= & \frac{-i B \delta_{m n}}{2} \int_{B}^{\infty} d t \frac{t-B}{t^{2}}\left[\frac{1}{t-\omega_{1}+\omega_{2}-2 i \epsilon}+\frac{1}{t-\omega_{2}+\omega_{1}-2 i \epsilon}\right] .
\end{aligned}
$$

This same integral appears in the other two diagrams, so we shall use this result again.

The first diagram has now been reduced to

$$
\begin{aligned}
& -2 i \delta_{r s} \int d \mathbf{y} d \mathbf{z} d \mathbf{u} d \mathbf{v} \int \frac{d \omega_{1}}{2 \pi} \frac{d \omega_{2}}{2 \pi} \\
& y^{j} \hat{G}_{\omega_{2}}(\mathbf{y}, \mathbf{u})\left(-i \nabla_{u}^{m}\right) \hat{G}_{\omega_{1}}(\mathbf{u}, \mathbf{z}) z^{k} \hat{G}_{\omega_{1}}(\mathbf{z}, \mathbf{v})\left(-i \nabla_{v}^{n}\right) \hat{G}_{\omega_{2}}(\mathbf{v}, \mathbf{y}) \\
& \frac{-i B \delta_{m n}}{2} \int_{B}^{\infty} d t \frac{t-B}{t^{2}}\left[\frac{1}{t-\omega_{1}+\omega_{2}-2 i \epsilon}+\frac{1}{t-\omega_{2}+\omega_{1}-2 i \epsilon}\right]
\end{aligned}
$$

Now consider separately the two terms in the last factor. In the first term close the $\omega_{1}$ integral in the upper half plane, but in the second close the $\omega_{2}$ integral in the upper half plane. In each case there is only a simple pole at $\omega_{1}=-B+i \epsilon$, respectively $\omega_{2}=-B+i \epsilon$. The potential double poles at these locations don't contribute because they correspond to two $s$-wave states separated by a vector operator. (This will not apply to the other two diagrams!) After this the remaining $\omega$ integral is done by again closing in the upper half plane. Altogether there are four separate contributions, each of which can be thought of as a ground state expectation value in the one body problem. The results of the steps described so 
far can be represented, in a self-explanatory notation, as

$$
\begin{aligned}
-B \delta_{r s} \int_{B}^{\infty} d t \frac{t-B}{t^{2}}[\langle B| & y^{k} \frac{1}{\mathbf{p}^{2}+B} p^{m} \hat{G}_{-t-B} y^{j} \frac{1}{\mathbf{p}^{2}+B+t} p^{m}|B\rangle \\
& \quad-\frac{1}{t}\left\langle B\left|y^{k} \frac{1}{\mathbf{p}^{2}+B} p^{m}\right| B\right\rangle\left\langle B\left|y^{j} \frac{1}{\mathbf{p}^{2}+B} p^{m}\right| B\right\rangle \\
+ & \left\langle B\left|p^{m} \frac{1}{\mathbf{p}^{2}+B+t} y^{j} \hat{G}_{-t-B} p^{m} \frac{1}{\mathbf{p}^{2}+B} y^{k}\right| B\right\rangle \\
& \quad-\frac{1}{t}\left\langle B\left|p^{m} \frac{1}{\mathbf{p}^{2}+B} y^{j}\right| B\right\rangle\left\langle B\left|p^{m} \frac{1}{\mathbf{p}^{2}+B} y^{k}\right| B\right\rangle \\
+\left\langle B\left|p^{m} \frac{1}{\mathbf{p}^{2}+B+t} y^{k} \hat{G}_{-t-B} p^{m} \frac{1}{\mathbf{p}^{2}+B} y^{j}\right| B\right\rangle & -\frac{1}{t}\left\langle B\left|p^{m} \frac{1}{\mathbf{p}^{2}+B} y^{k}\right| B\right\rangle\left\langle B\left|p^{m} \frac{1}{\mathbf{p}^{2}+B} y^{j}\right| B\right\rangle \\
+ & \left.\quad y^{j} \frac{1}{\mathbf{p}^{2}+B} p^{m} \hat{G}-t-B y^{k} \frac{1}{\mathbf{p}^{2}+B+t} p^{m}|B\rangle\right] \\
& \left.-\frac{1}{t}\left\langle B\left|y^{j} \frac{1}{\mathbf{p}^{2}+B} p^{m}\right| B\right\rangle\left\langle B\left|y^{k} \frac{1}{\mathbf{p}^{2}+B} p^{m}\right| B\right\rangle\right]
\end{aligned}
$$

where we have replaced the first and last Green functions in each term by the free ones, since the $s$-wave parts decouple in these locations. We postpone explicit evaluation until we have reduced the remaining diagrams. In addition, $\epsilon$ may be safely set to zero in all of the terms in (4.11).

We present the expressions for the second and third diagrams of Fig. 3 after the first simplification step analogous to (4.10). The second diagram leads to

$$
\begin{aligned}
& -2 i \delta_{r s} \int d \mathbf{y} d \mathbf{z} d \mathbf{u} d \mathbf{v} \int \frac{d \omega_{1}}{2 \pi} \frac{d \omega_{2}}{2 \pi} \\
& y^{j} \hat{G}_{\omega_{1}}(\mathbf{y}, \mathbf{z}) z^{k} \hat{G}_{\omega_{1}}(\mathbf{z}, \mathbf{u})\left(-i \nabla_{u}^{m}\right) \hat{G}_{\omega_{2}}(\mathbf{u}, \mathbf{v})\left(-i \nabla_{v}^{m}\right) \hat{G}_{\omega_{1}}(\mathbf{v}, \mathbf{y}) \\
& \frac{-i B}{2} \int_{B}^{\infty} d t \frac{t-B}{t^{2}}\left[\frac{1}{t-\omega_{1}+\omega_{2}-2 i \epsilon}+\frac{1}{t-\omega_{2}+\omega_{1}-2 i \epsilon}\right]
\end{aligned}
$$


and the third to

$$
\begin{aligned}
& -2 i \delta_{r s} \int d \mathbf{y} d \mathbf{z} d \mathbf{u} d \mathbf{v} \int \frac{d \omega_{1}}{2 \pi} \frac{d \omega_{2}}{2 \pi} \\
& y^{j} \hat{G}_{\omega_{1}}(\mathbf{y}, \mathbf{u})\left(-i \nabla_{u}^{m}\right) \hat{G}_{\omega_{2}}(\mathbf{u}, \mathbf{v})\left(-i \nabla_{v}^{m}\right) \hat{G}_{\omega_{1}}(\mathbf{v}, \mathbf{z}) z^{k} \hat{G}_{\omega_{1}}(\mathbf{z}, \mathbf{y}) \\
& \frac{-i B}{2} \int_{B}^{\infty} d t \frac{t-B}{t^{2}}\left[\frac{1}{t-\omega_{1}+\omega_{2}-2 i \epsilon}+\frac{1}{t-\omega_{2}+\omega_{1}-2 i \epsilon}\right] .
\end{aligned}
$$

The next step is to consider separately the expressions arising from the two terms in the last factor. For the first term, close the $\omega_{1}$ contour up and for the second close the $\omega_{2}$ contour up. Here the asymmetry between $\omega_{1}$ and $\omega_{2}$ causes some differences from the corresponding simplification of the first diagram. The first terms involve both a double pole and and three simple poles at $\omega_{1}=-B+i \epsilon$. Whereas the second terms involve only a single simple pole. The four simple pole contributions from each diagram lead to expressions very like (4.11). Namely,

$$
\begin{aligned}
-B \delta_{r s} \int_{B}^{\infty} d t \frac{t-B}{t^{2}}\left[\left\langle B\left|y^{k} \frac{1}{\mathbf{p}^{2}+B} p^{m} \hat{G}_{-t-B} p^{m} \frac{1}{\mathbf{p}^{2}+B} y^{j}\right| B\right\rangle\right. \\
\quad-\frac{1}{t}\left\langle B\left|y^{k} \frac{1}{\mathbf{p}^{2}+B} p^{m}\right| B\right\rangle\left\langle B\left|p^{m} \frac{1}{\mathbf{p}^{2}+B} y^{j}\right| B\right\rangle \\
+\left\langle B\left|p^{m} \frac{1}{\mathbf{p}^{2}+B+t} p^{m} \tilde{G}_{-B} y^{j} \frac{1}{\mathbf{p}^{2}+B} y^{k}\right| B\right\rangle \\
+\left\langle B\left|p^{m} \frac{1}{\mathbf{p}^{2}+B+t} y^{j} \hat{G}_{-t-B} y^{k} \frac{1}{\mathbf{p}^{2}+B+t} p^{m}\right| B\right\rangle \\
\quad-\frac{1}{t}\left\langle B\left|p^{m} \frac{1}{\mathbf{p}^{2}+B} y^{j}\right| B\right\rangle\left\langle B\left|y^{k} \frac{1}{\mathbf{p}^{2}+B} p^{m}\right| B\right\rangle \\
\left.+\left\langle B\left|y^{j} \frac{1}{\mathbf{p}^{2}+B} y^{k} \tilde{G}_{-B} p^{m} \frac{1}{\mathbf{p}^{2}+B+t} p^{m}\right| B\right\rangle\right]
\end{aligned}
$$


for the first diagram, and

$$
\begin{aligned}
-B \delta_{r s} \int_{B}^{\infty} d t \frac{t-B}{t^{2}}[ & \left\langle B\left|y^{k} \frac{1}{\mathbf{p}^{2}+B} y^{j} \tilde{G}_{-B} p^{m} \frac{1}{\mathbf{p}^{2}+B+t} p^{m}\right| B\right\rangle \\
& +\left\langle B\left|p^{m} \frac{1}{\mathbf{p}^{2}+B+t} p^{m} \tilde{G}_{-B} y^{k} \frac{1}{\mathbf{p}^{2}+B} y^{j}\right| B\right\rangle \\
& +\left\langle B\left|p^{m} \frac{1}{\mathbf{p}^{2}+B+t} y^{k} \hat{G}_{-t-B} y^{j} \frac{1}{\mathbf{p}^{2}+B+t} p^{m}\right| B\right\rangle \\
& -\frac{1}{t}\left\langle B\left|p^{m} \frac{1}{\mathbf{p}^{2}+B} y^{k}\right| B\right\rangle\left\langle B\left|y^{j} \frac{1}{\mathbf{p}^{2}+B} p^{m}\right| B\right\rangle \\
& \left.+\left\langle B\left|y^{j} \frac{1}{\mathbf{p}^{2}+B} p^{m} \hat{G}_{-t-B} p^{m} \frac{1}{\mathbf{p}^{2}+B} y^{k}\right| B\right\rangle\right] \\
& \left.\quad-\frac{1}{t}\left\langle B\left|y^{j} \frac{1}{\mathbf{p}^{2}+B} p^{m}\right| B\right\rangle\left\langle B\left|p^{m} \frac{1}{\mathbf{p}^{2}+B} y^{k}\right| B\right\rangle\right]
\end{aligned}
$$

for the second. (4.14) and (4.15) do not include the double pole contribution to the integral of the first terms over $\omega_{1}$. These arise from the ground state contributions to the second and fourth $\hat{G}$ 's in (4.12) and to the first and third $\hat{G}$ 's in (4.13). Evaluating the residue of the double pole in $\omega_{1}$ and then doing the $\omega_{2}$ integral leads to the double pole contributions

$$
\begin{gathered}
B \delta_{r s} \int_{B}^{\infty} d t \frac{t-B}{t^{2}} \frac{d}{d \omega}\left[\left\langle B\left|y^{j} \frac{1}{\mathbf{p}^{2}+B-\omega} y^{k}\right| B\right\rangle\left\langle B\left|p^{m} \frac{1}{\mathbf{p}^{2}+B+t-\omega} p^{m}\right| B\right\rangle\right]_{\omega=0} \\
=B \delta_{r s} \int_{B}^{\infty} d t \frac{t-B}{t^{2}}\left[\left\langle B\left|y^{j} \frac{1}{\left(\mathbf{p}^{2}+B\right)^{2}} y^{k}\right| B\right\rangle\left\langle B\left|p^{m} \frac{1}{\mathbf{p}^{2}+B+t} p^{m}\right| B\right\rangle\right. \\
\left.\quad+\left\langle B\left|y^{j} \frac{1}{\mathbf{p}^{2}+B} y^{k}\right| B\right\rangle\left\langle B\left|p^{m} \frac{1}{\left(\mathbf{p}^{2}+B+t\right)^{2}} p^{m}\right| B\right\rangle\right]
\end{gathered}
$$


and

$$
\begin{aligned}
B \delta_{r s} \int_{B}^{\infty} d t \frac{t-B}{t^{2}} \frac{d}{d \omega}\left[\left\langle B\left|y^{k} \frac{1}{\mathbf{p}^{2}+B-\omega} y^{j}\right| B\right\rangle\left\langle B\left|p^{m} \frac{1}{\mathbf{p}^{2}+B+t-\omega} p^{m}\right| B\right\rangle\right]_{\omega=0} \\
=B \delta_{r s} \int_{B}^{\infty} d t \frac{t-B}{t^{2}}\left[\left\langle B\left|y^{k} \frac{1}{\left(\mathbf{p}^{2}+B\right)^{2}} y^{j}\right| B\right\rangle\left\langle B\left|p^{m} \frac{1}{\mathbf{p}^{2}+B+t} p^{m}\right| B\right\rangle\right. \\
\left.\quad+\left\langle B\left|y^{k} \frac{1}{\mathbf{p}^{2}+B} y^{j}\right| B\right\rangle\left\langle B\left|p^{m} \frac{1}{\left(\mathbf{p}^{2}+B+t\right)^{2}} p^{m}\right| B\right\rangle\right]
\end{aligned}
$$

respectively.

Notice that lines 2 and 5 of (4.14) and lines 4 and 6 of (4.15) cancel lines 2, 4, 6 , and 8 of (4.11) by virtue of the fact that

$$
\left\langle B\left|y^{j} \frac{1}{\mathbf{p}^{2}+B} p^{m}\right| B\right\rangle=-\left\langle B\left|p^{m} \frac{1}{\mathbf{p}^{2}+B} y^{j}\right| B\right\rangle
$$

by time reversal invariance. This cancellation together with similarity of structure make it helpful to regroup the various contributions by gathering together all terms with the central Green function $\hat{G}_{-t-B}$ in group 1 ,

$$
\begin{aligned}
-B \delta_{r s} \int_{B}^{\infty} d t \frac{t-B}{t^{2}}[ & \left\langle B\left|y^{k} \frac{1}{\mathbf{p}^{2}+B} p^{m} \hat{G}_{-t-B} y^{j} \frac{1}{\mathbf{p}^{2}+B+t} p^{m}\right| B\right\rangle \\
& +\left\langle B\left|p^{m} \frac{1}{\mathbf{p}^{2}+B+t} y^{j} \hat{G}_{-t-B} p^{m} \frac{1}{\mathbf{p}^{2}+B} y^{k}\right| B\right\rangle \\
& +\left\langle B\left|p^{m} \frac{1}{\mathbf{p}^{2}+B+t} y^{k} \hat{G}_{-t-B} p^{m} \frac{1}{\mathbf{p}^{2}+B} y^{j}\right| B\right\rangle \\
& \left.+\left\langle B\left|y^{j} \frac{1}{\mathbf{p}^{2}+B} p^{m} \hat{G}_{-t-B} y^{k} \frac{1}{\mathbf{p}^{2}+B+t} p^{m}\right| B\right\rangle\right] \\
& +\left\langle B\left|y^{k} \frac{1}{\mathbf{p}^{2}+B} p^{m} \hat{G}_{-t-B} p^{m} \frac{1}{\mathbf{p}^{2}+B} y^{j}\right| B\right\rangle \\
& +\left\langle B\left|p^{m} \frac{1}{\mathbf{p}^{2}+B+t} y^{j} \hat{G}_{-t-B} y^{k} \frac{1}{\mathbf{p}^{2}+B+t} p^{m}\right| B\right\rangle \\
& +\left\langle B\left|p^{m} \frac{1}{\mathbf{p}^{2}+B+t} y^{k} \hat{G}_{-t-B} y^{j} \frac{1}{\mathbf{p}^{2}+B+t} p^{m}\right| B\right\rangle \\
& \left.+\left\langle B\left|y^{j} \frac{1}{\mathbf{p}^{2}+B} p^{m} \hat{G}_{-t-B} p^{m} \frac{1}{\mathbf{p}^{2}+B} y^{k}\right| B\right\rangle\right],
\end{aligned}
$$


and all those with central Green function $\tilde{G}_{-B}$ into group 2,

$$
\begin{aligned}
-B \delta_{r s} \int_{B}^{\infty} d t \frac{t-B}{t^{2}}[ & \left\langle B\left|p^{m} \frac{1}{\mathbf{p}^{2}+B+t} p^{m} \tilde{G}_{-B} y^{j} \frac{1}{\mathbf{p}^{2}+B} y^{k}\right| B\right\rangle \\
& +\left\langle B\left|y^{j} \frac{1}{\mathbf{p}^{2}+B} y^{k} \tilde{G}_{-B} p^{m} \frac{1}{\mathbf{p}^{2}+B+t} p^{m}\right| B\right\rangle \\
& +\left\langle B\left|y^{k} \frac{1}{\mathbf{p}^{2}+B} y^{j} \tilde{G}_{-B} p^{m} \frac{1}{\mathbf{p}^{2}+B+t} p^{m}\right| B\right\rangle \\
& \left.+\left\langle B\left|p^{m} \frac{1}{\mathbf{p}^{2}+B+t} p^{m} \tilde{G}_{-B} y^{k} \frac{1}{\mathbf{p}^{2}+B} y^{j}\right| B\right\rangle\right] .
\end{aligned}
$$

The double pole contributions are also naturally associated with group 2 .

In the appendix we describe the detailed evaluation of all these expressions. By far the largest contribution comes from the second and third diagrams of Fig. 3. The total contribution of the first diagram is approximately $-0.055197 / B^{2}$ or about $16.6 \%$ of the random phase approximation. Compare this to the total contribution of the second and third diagrams of $+0.50913 / B^{2}$ or $-153 \%$ of the RPA. In the next section we show that this huge correction can be attributed to a substantial shift in the ionization energy of the open polymer. The large terms in perturbation theory can be identified as "self energy" corrections which can be easily summed as a geometric series in the usual way.

\section{Summing Large Corrections}

We have found very large corrections to RPA in next to lowest order of perturbation theory. But we have also seen that all the large contributions come from diagrams that have the interpretation as self energy bubbles on the propagators entering the RPA. Thus we can hope that an improved RPA would result if we use the full propagators rather than the bare ones. In other words, we should take the sum of diagrams shown in Fig. 4 as the propagator to use in RPA. An even more sophisticated improvement would be to replace each bubble in Fig. 4 with the RPA sum of Fig. 2. 
FIG. 4. Summing self energy diagrams.

More generally we can define a "self energy" part, $\Sigma$, so that $i \Sigma$ is the sum of all the (amputated) one particle irreducible diagrams contributing to the correlation function of $\psi$ and $\psi^{\dagger}$. Then the full propagator

$$
\left\langle G\left|T\left[\psi_{r}(\mathbf{y}, t) \psi_{s}^{\dagger}(\mathbf{z}, 0)\right]\right| G\right\rangle \equiv-i \delta_{r, s} \Delta
$$

satisfies an integral equation

$$
\Delta=\hat{G}+\hat{G} \Sigma \Delta
$$

Because the operator $\psi_{r}$ annihilates a bond in the polymer, the location in $-\omega$ of the lowest pole in $\Delta$ gives the "exact" ionization energy, which in zeroth order of perturbation theory is just $B$, the lowest pole in $\hat{G}$. To find this lowest pole it is sufficient to look at a matrix element of $\Delta$ between any two states which couple to it. Assuming that the zeroth order ground state continues to couple to this pole, it is convenient to use a new concept of "irreducible" to mean that one can't cut the diagram in two by cutting a single line in its ground state. This new irreducible part will then be called $\Sigma^{\prime}$. A formula for $\Sigma^{\prime}$ is obtained by splitting $\hat{G}=\tilde{G}+G_{0}$ (see (4.8)) where, in frequency space,

$$
G_{0} \equiv \frac{|B\rangle\langle B|}{-B-\omega+i \epsilon}
$$

Then we have the integral equation

$$
\Sigma^{\prime}=\Sigma+\Sigma \tilde{G} \Sigma^{\prime}
$$


That is, we put back into $\Sigma^{\prime}$ all those reducible contributions for which the single line is in an excited state.

With these definitions a little algebraic gymnastics leads to the following formula for the full propagator:

$$
\Delta=\tilde{G}+\tilde{G} \Sigma^{\prime} \tilde{G}+\left(I+\tilde{G} \Sigma^{\prime}\right) \frac{1}{-B-\omega-\Sigma_{0}^{\prime}+i \epsilon} P_{0}\left(I+\Sigma^{\prime} \tilde{G}\right)
$$

where $P_{0} \equiv|B\rangle\langle B|$ is the projector onto the zeroth order ground state, and $\Sigma_{0}^{\prime} \equiv$ $\left\langle B\left|\Sigma^{\prime}\right| B\right\rangle$ a real number. As we explain in the Appendix, the first step in the evaluation of a typical diagram is to close one or more of the frequency integrals in the upper half plane, picking up some of the ground state poles in the various $\hat{G}_{\omega}$ 's. In a more sophisticated application of perturbation theory, where $\Delta$ is used in place of $\hat{G}$, this step will pick up the pole at $\omega=\omega_{0}$ where

$$
D\left(\omega_{0}\right) \equiv-B-\omega_{0}-\Sigma_{0}^{\prime}\left(\omega_{0}\right)+i \epsilon=0
$$

The residue of this pole is

$$
\frac{1}{D^{\prime}\left(\omega_{0}\right)}\left(I+\tilde{G}_{\omega_{0}} \Sigma^{\prime}\left(\omega_{0}\right)\right) P_{0}\left(I+\Sigma^{\prime}\left(\omega_{0}\right) \tilde{G}_{\omega_{0}}\right)=\frac{1}{D^{\prime}\left(\omega_{0}\right)} G_{\omega_{0}} \Sigma^{\prime}\left(\omega_{0}\right) P_{0} \Sigma^{\prime}\left(\omega_{0}\right) G_{\omega_{0}}
$$

Note that the form on the r.h.s. would be meaningless if $\omega_{0}=B$ since $G_{-B}$ is infinite. But since the shift of $\omega_{0}$ away from its zeroth order value is taken into account, this is not a problem. (If by some fluke the shift were zero, then $\Sigma_{-B}^{\prime}$ would supply a zero multiplying the infinity, leaving the r.h.s. indeterminate.)

Now we can use these results to specify our improved version of RPA. It is obtained by replacing each of the bare propagators in the first diagram of Fig. 2 with the the full propagator (5.3). Corrections which involve "self energy" insertions such as the second and third diagrams of Fig. 3, will then automatically be included in this approximation. The first diagram of Fig. 3 is a typical diagram not included. Our experience with straight perturbation theory indicates that the diagrams not included give roughly a $15 \%$ correction. 
Inserting (5.3) into the RPA diagram, we can carry out the $\omega$ integral, just as with the bare propagators, by closing the contour into the upper half plane, picking up the lowest pole at $\omega=\omega_{0}$, determined by (5.4). The $+i \epsilon$ of (5.4) amounts to the contour prescription which states that this lowest pole is the only singularity picked up by this procedure. The result is

$$
\Pi_{y y, r s}^{\operatorname{Improved}, j k}(0)=-2 \delta_{r s} \frac{\left\langle B\left|\Sigma^{\prime}\left(\omega_{0}\right) G_{\omega_{0}} y^{j}\left[\tilde{G}_{\omega_{0}}+\tilde{G}_{\omega_{0}} \Sigma^{\prime}\left(\omega_{0}\right) \tilde{G}_{\omega_{0}}\right] y^{k} G_{\omega_{0}} \Sigma^{\prime}\left(\omega_{0}\right)\right| B\right\rangle}{1+\left.\frac{d}{d \omega} \Sigma_{0}^{\prime}(\omega)\right|_{\omega=\omega_{0}}} .
$$

Note that the pole contribution to $\Delta$ vanishes when sandwiched between $y^{j}$ and $y^{k}$ by rotational invariance. If we were given the exact result for $\Sigma^{\prime}$ we could use this formula to calculate the string tension. We would first have to find $\omega_{0}$ by solving (5.4). Then evaluating $\Sigma^{\prime}$ at $\omega_{0}$ and plugging into (5.6) would give us the required information.

Unfortunately, $\Sigma^{\prime}$ is not a quantity we can evaluate exactly, and to assess the ramifications of this procedure we must make further approximations. A plausible approximation would be to calculate $\Sigma$ in perturbation theory. Thus in lowest order we would simply take the second diagram of Fig. 4, with external propagators removed, for $\Sigma(\omega)$. One could then try to use this approximate form for $\Sigma$ in (5.2), to determine $\Sigma^{\prime}$. Although inexact, this is a substantial improvement over straight perturbation theory because, for instance, the procedure does not violate easily proved positivity constraints on $\mathcal{G}_{r s}$. Probably the approximation most consistent with RPA would be to use the RPA sum for the bubble in the lowest order diagram for $\Sigma$, as we described in the opening paragraph of this section. In the remainder of this section we attempt a simplified version of the first mentioned scheme. The complete implementations of the first scheme and of the second would involve some nontrivial technical developments, and we leave them for future work.

To get a feeling for how these considerations affect the numerical results of the previous section, we close this section with the evaluation of the string tension 
using the lowest order approximation to $\Sigma$,

$$
\begin{aligned}
& \Sigma(\omega) \approx \\
& \frac{B}{\pi} \int d \mathbf{q} \frac{\mathbf{q}^{2}}{\left(\mathbf{q}^{2}+B\right)^{2}}\left(\sum_{m>0} \frac{p^{k}|m\rangle\langle m| p^{k}}{E_{m}+\mathbf{q}^{2}+B-\omega-3 i \epsilon}+\frac{p^{k}|0\rangle\langle 0| p^{k}}{E_{0}+\mathbf{q}^{2}+B-\omega+3 i \epsilon}\right),
\end{aligned}
$$

in an approximate version of (5.2). Recalling that the large corrections found in the previous section could be traced to the contribution of the second term of (4.7), it would seem to be reasonable to truncate the $\tilde{G}$ appearing in (5.2) to that term. We therefore define, in notation explained in the appendix,

$$
\tilde{G} \approx \tilde{G}_{\text {trunc }}(\omega) \equiv \frac{1}{B \ln (-\omega / B)}|-\omega\rangle\left\langle-\omega\left|-\frac{1}{-\omega-B}\right| B\right\rangle\langle B|
$$

We can then use (5.2) to determine by straightforward algebra the matrix elements $\left\langle B\left|\Sigma^{\prime}\right| B\right\rangle,\left\langle-\omega\left|\Sigma^{\prime}\right| B\right\rangle,\left\langle B\left|\Sigma^{\prime}\right|-\omega\right\rangle$, and $\left\langle-\omega\left|\Sigma^{\prime}\right|-\omega\right\rangle$ in terms of analogous matrix elements of $\Sigma$.

Define $\gamma_{\omega} \equiv 1 /\{B \ln (-\omega / B)\}, \gamma_{0} \equiv 1 /(\omega+B)$, and

$$
\begin{aligned}
D_{1} \equiv 1- & \gamma_{\omega}\langle-\omega|\Sigma|-\omega\rangle-\gamma_{0}\langle B|\Sigma| B\rangle+ \\
& \gamma_{\omega} \gamma_{0}[\langle B|\Sigma| B\rangle\langle-\omega|\Sigma|-\omega\rangle-\langle-\omega|\Sigma| B\rangle\langle B|\Sigma|-\omega\rangle] .
\end{aligned}
$$

Then we find

$$
\begin{aligned}
\left\langle B\left|\Sigma^{\prime}\right| B\right\rangle & \approx \frac{\langle B|\Sigma| B\rangle-\gamma_{\omega}[\langle B|\Sigma| B\rangle\langle-\omega|\Sigma|-\omega\rangle-\langle-\omega|\Sigma| B\rangle\langle B|\Sigma|-\omega\rangle]}{D_{1}} \\
\left\langle-\omega\left|\Sigma^{\prime}\right| B\right\rangle & \approx \frac{\langle-\omega|\Sigma| B\rangle}{D_{1}} \\
\left\langle B\left|\Sigma^{\prime}\right|-\omega\right\rangle & \approx \frac{\langle B|\Sigma|-\omega\rangle}{D_{1}} \\
\left\langle-\omega\left|\Sigma^{\prime}\right|-\omega\right\rangle & \approx \\
& \frac{\langle-\omega|\Sigma|-\omega\rangle-\gamma_{0}[\langle B|\Sigma| B\rangle\langle-\omega|\Sigma|-\omega\rangle-\langle-\omega|\Sigma| B\rangle\langle B|\Sigma|-\omega\rangle]}{D_{1}}
\end{aligned}
$$

For the matrix elements of $\Sigma$, we find, after doing angular integrals and some minor 
changes of integration variables,

$$
\begin{aligned}
\langle B|\Sigma| B\rangle & =B \int_{1}^{\infty} \frac{d v}{v^{2}} \int_{1}^{\infty} \frac{d u}{u^{2}} \frac{(v-1)(u-1)}{u+v-1-\omega / B} \equiv B f_{1}(\rho) \\
\langle B|\Sigma|-\omega\rangle & =B \int_{1}^{\infty} \frac{d v}{v^{2}} \int_{1}^{\infty} \frac{d u}{u} \frac{(v-1)(u-1)}{(u-1-\omega / B)(u+v-1-\omega / B)} \equiv B f_{2}(\rho) \\
\langle-\omega|\Sigma| B\rangle & =\langle B|\Sigma|-\omega\rangle=B f_{2}(\rho) \\
\langle-\omega|\Sigma|-\omega\rangle & =B \int_{1}^{\infty} \frac{d v}{v^{2}} \int_{1}^{\infty} \frac{d u}{(u-1-\omega / B)^{2}} \frac{(v-1)(u-1)}{u+v-1-\omega / B} \equiv B f_{3}(\rho)
\end{aligned}
$$

where we have defined $\rho \equiv-1-\omega / B$. The $v$ integral is elementary, with the results

$$
\begin{aligned}
& f_{1}(\rho)=\int_{1}^{\infty} \frac{(u-1) d u}{u^{2}}\left[\frac{\ln (1+u+\rho)-1}{u+\rho}+\frac{\ln (1+u+\rho)}{(u+\rho)^{2}}\right] \\
& f_{2}(\rho)=\int_{1}^{\infty} \frac{(u-1) d u}{u}\left[\frac{\ln (1+u+\rho)-1}{(u+\rho)^{2}}+\frac{\ln (1+u+\rho)}{(u+\rho)^{3}}\right] \\
& f_{3}(\rho)=\int_{1}^{\infty}(u-1) d u\left[\frac{\ln (1+u+\rho)-1}{(u+\rho)^{3}}+\frac{\ln (1+u+\rho)}{(u+\rho)^{4}}\right] .
\end{aligned}
$$

Putting everything together, we express all quantities in terms of the functions $f_{1}, f_{2}, f_{3}$. For $D_{1}$ we have

$$
D_{1} \approx 1-\frac{f_{3}(\rho)}{\ln (1+\rho)}+\frac{f_{1}(\rho)}{\rho}-\frac{f_{1}(\rho) f_{3}(\rho)-f_{2}(\rho)^{2}}{\rho \ln (1+\rho)}
$$


and for the matrix elements of $\Sigma^{\prime}$,

$$
\begin{aligned}
\left\langle B\left|\Sigma^{\prime}\right| B\right\rangle & \approx B \frac{f_{1}(\rho)-\left[f_{1}(\rho) f_{3}(\rho)-f_{2}(\rho)^{2}\right] / \ln (1+\rho)}{D_{1}} \\
\left\langle-\omega\left|\Sigma^{\prime}\right| B\right\rangle & \approx B \frac{f_{2}(\rho)}{D_{1}} \\
\left\langle B\left|\Sigma^{\prime}\right|-\omega\right\rangle & \approx B \frac{f_{2}(\rho)}{D_{1}} \\
\left\langle-\omega\left|\Sigma^{\prime}\right|-\omega\right\rangle & \approx B \frac{f_{3}(\rho)+\left[f_{1}(\rho) f_{3}(\rho)-f_{2}(\rho)^{2}\right] / \rho}{D_{1}} .
\end{aligned}
$$

Using the above approximate result for $\left\langle B\left|\Sigma^{\prime}\right| B\right\rangle=\Sigma_{0}^{\prime}$ in (5.4) gives the following approximate equation for $\rho_{0}=-1-\omega_{0} / B$ :

$$
\rho_{0}-\frac{f_{1}\left(\rho_{0}\right)-\left[f_{1}\left(\rho_{0}\right) f_{3}\left(\rho_{0}\right)-f_{2}\left(\rho_{0}\right)^{2}\right] / \ln \left(1+\rho_{0}\right)}{D_{1}\left(\rho_{0}\right)} \approx 0 .
$$

Using a simple iterative search for solutions to this equation leads to a value for $\rho_{0}$ between 0.589941 and 0.589942 , so we can say that $\rho_{0} \approx 0.590$. This corresponds to a shift in the ionization energy from $B$ to $1.590 B$. The same numerical data give $1+d \Sigma_{0}^{\prime} / d \omega \approx 1.06$, which is consistent with unity in the present discussion. Straight perturbation theory would lead to the result $\omega_{0}=-\left(1+f_{1}(0)\right) B$. But $f_{1}(0)=I_{11}-I_{21} \approx 0.59086$. So, curiously enough, the improved prediction for $\omega_{0}$ is barely distinguishable from the calculation of the first two orders of perturbation theory! As an aside, we recall that in the Hartree-Fock method which we are using here, the relationship of the shift in ionization energy (or single particle energy) is related to the shift in the ground state energy per bond (or per single particle) by a factor of 2 . Thus this $60 \%$ shift in the ionization energy, which is rather huge, corresponds to a more moderate $30 \%$ shift in the ground state energy.

Having approximately determined $\omega_{0}$, we consider the evaluation of (5.6) at this value. In the same spirit as our approximate treatment of (5.2), It is reasonable to approximate the first and last $G_{\omega_{0}}$ 's on the r.h.s. by

$$
G_{\omega_{0}} \approx \frac{1}{B \ln \left(-\omega_{0} / B\right)}\left|-\omega_{0}\right\rangle\left\langle-\omega_{0}\right|
$$


Then

$$
\begin{aligned}
& \Pi_{y y, r s}^{\operatorname{Improved}, j k}(0) \approx \\
& -2 \delta_{r s} \frac{\left|\left\langle B\left|\Sigma^{\prime}\left(\omega_{0}\right)\right|-\omega_{0}\right\rangle\right|^{2}}{\left(1+\frac{d}{d \omega} \Sigma_{0}^{\prime}(\omega) \mid \omega=\omega_{0}\right) \ln ^{2}\left(1+\rho_{0}\right)}\left\langle-\omega_{0}\left|y^{j}\left[\tilde{G}_{\omega_{0}}+\tilde{G}_{\omega_{0}} \Sigma^{\prime}\left(\omega_{0}\right) \tilde{G}_{\omega_{0}}\right] y^{k}\right|-\omega_{0}\right\rangle
\end{aligned}
$$

Referring to our experience with straight perturbation theory the second term includes contributions in next to lowest order which are relatively small, i.e. roughly $15 \%$. Thus it is fair to drop it in the present approximation. The resulting matrix element is easily evaluated

$$
\left\langle-\omega_{0}\left|y^{j} \tilde{G}_{\omega_{0}} y^{k}\right|-\omega_{0}\right\rangle=\frac{\delta_{j k}}{6 B^{2}\left(1+\rho_{0}\right)^{3}} \approx 0.249 \frac{\delta_{j k}}{6 B^{2}}
$$

Thus to an expected $15 \%$ accuracy we have

$$
\Pi_{y y, r s}^{\operatorname{Improved}, j k}(0) \approx-2 \delta_{r s} \frac{f_{2}\left(\rho_{0}\right)^{2}}{D_{1}\left(\rho_{0}\right)^{2} \ln ^{2}\left(1+\rho_{0}\right)}\left\langle-\omega_{0}\left|y^{j} \tilde{G}_{\omega_{0}} y^{k}\right|-\omega_{0}\right\rangle \approx-0.333 \frac{\delta_{r s} \delta_{j k}}{3 B^{2}} .
$$

Thus our improved RPA causes predicts a reduction in $\Pi$ by approximately a factor of 3 over the näive RPA. But now we have explicitly incorporated all of the large corrections in next order in a partial resummation of perturbation theory. The effects left out amount to a roughly $15 \%$ correction in this next order, and it is reasonable to expect that this is a rough indication of the accuracy of our improved RPA. The factor of 3 reduction in $\Pi$ corresponds to a factor of $\sqrt{3}$ increase in the rest tension leading to

$$
T_{0}^{\text {Improved RPA }}=\frac{1}{\alpha^{\text {Improved RPA }}} \approx 3 B
$$




\section{Conclusions}

In this article we have initiated a detailed quantitative study of the "wee parton" formulation of string theory proposed in Ref.[3,9]. Although the quantitative relationship between the microscopic string bit dynamics and the low energy stringy

properties of string theory is subsumed in merely a renormalization of the string tension, we believe that these details are extremely important for understanding any processes which are capable of probing the microscopic structure of the theory. A gedanken experiment which could in principle reveal this structure might be the physics near the horizon of a black hole as perceived by an observer far away. ${ }^{[16-18]}$ It is, of course, unlikely that the specific string bit model we have chosen to analyze in this article is the ultimate underlying dynamics of the bosonic string. Our purpose, rather, is to pick a model and explore its detailed dynamics using well-known methods in many body physics. The model we have chosen, with its delta function interaction potential, does nonetheless have several appealing features which recommend it, including locality in the transverse space and (anomalous) scale invariance.

For pedagogical simplicity we have only tried to account for the bosonic string in this article. But one reason for subjecting the string bit dynamics for this unrealistic model to such scrutiny is that the superstring could be much more sensitive to the microscopic details than is the bosonic string. In that case we need to have a high degree of confidence in the techniques used to analyze a superstring bit model. We hope to devise such a model in the near future.

The numerical results of our study are encouraging. Although the most nä̈ve application of RPA to the calculation of the rest tension is subjected to huge $(150 \%)$ corrections in next order of perturbation theory, we have shown that the bulk of these corrections can be incorporated in a Hartree-Fock like improvement of the zeroth order RPA. The corrections that remain are estimated to be around 15\%. We did make justifiable simplifications of the Hartree-Fock treatment, but there is certainly room for improvement. It would be particularly desirable to go 
beyond the lowest order approximation for $\Sigma$. Also, in calculating $\Sigma^{\prime}$ we truncated the Green function $G^{\prime}$ by dropping the "free" contribution which was shown to be relatively small in perturbation theory. However, it should be a tractable problem without this truncation. Thus, although the simplifications we made were consistent with our approximation, we expect that they can be avoided with a little more effort.

Acknowledgements. The older work reviewed in this article and published in Ref.[3] benefited from valuable discussions with Jeffrey Goldstone. I thank him again for his direct contributions, cited in the text, as well as for his insights and advice on theoretical methods in many body physics. I also thank the Aspen Center for Physics where the manuscript for this article was completed.

\section{APPENDIX}

In this appendix, we describe the evaluation of the various matrix elements and integrals occurring in (4.18) and (4.19). The Green function occurring in group 1 is not singular in the integration range, so all $\epsilon$ 's may be set to zero. Then there is no distinction between $\hat{G}$ and $G$, so referring to (4.7) and going to momentum representation, we may write

$$
\hat{G}_{-t-B}=\frac{1}{\mathbf{p}^{2}+B+t}+\frac{|t+B\rangle\langle t+B|}{B \ln (1+t / B)},
$$

where we have introduced the state $|t+B\rangle$ defined by

$$
\langle\mathbf{p} \mid t+B\rangle \equiv \sqrt{\frac{B}{\pi}} \frac{1}{\mathbf{p}^{2}+B+t} .
$$

Defining

$$
\left.\left|B^{\prime}\right\rangle \equiv \frac{d}{d t}|t+B\rangle\right|_{t=0},
$$


we can also write

$$
\tilde{G}_{-B}=\frac{1}{\mathbf{p}^{2}+B}+\frac{1}{2 B}|B\rangle\left\langle B|+| B^{\prime}\right\rangle\langle B|+| B\rangle\left\langle B^{\prime}\right|
$$

We first consider the contributions due to the first terms in (A.1) and (A.2), the "free" Green functions. The first four matrix elements in (4.18) turn out to equal each other. Taking the first one, for example, we get

$$
\begin{aligned}
& \frac{B}{\pi} \int d \mathbf{p} \frac{2 i p^{k} p^{m}}{\left(\mathbf{p}^{2}+B\right)^{3}} \frac{1}{\left(\mathbf{p}^{2}+B+t\right)^{2}} i \frac{\partial}{\partial p^{j}} \frac{p^{m}}{\left(\mathbf{p}^{2}+B+t\right)\left(\mathbf{p}^{2}+B\right)}= \\
& \delta_{k j} B \int_{B}^{\infty} d u\left\{\left[\frac{2 B^{2}}{u^{4}}-\frac{4 B}{u^{3}}+\frac{2}{u^{2}}\right] \frac{1}{(u+t)^{3}}+\left[\frac{2 B^{2}}{u^{5}}-\frac{3 B}{u^{4}}+\frac{1}{u^{3}}\right] \frac{1}{(u+t)^{2}}\right\}
\end{aligned}
$$

Let us define

$$
I_{n, m} \equiv \int_{1}^{\infty} d v \frac{v-1}{v^{2}} \int_{1}^{\infty} d u \frac{1}{u^{n}(u+v)^{m}}
$$

Needed numerical values for some of the $I_{n, m}$ are displayed in the following table.

\begin{tabular}{|l|c|c|c|c|c|}
\hline \multicolumn{6}{|c|}{$I_{n, m}$} \\
\hline $\mathrm{n} \backslash \mathrm{m}$ & 1 & 2 & 3 & 4 & 5 \\
\hline 0 & & & & 0.007530 & 0.001851 \\
\hline 1 & 0.886294 & 0.090863 & 0.017005 & 0.004129 & 0.001163 \\
\hline 2 & 0.295431 & 0.045431 & 0.010202 & 0.002751 & 0.000832 \\
\hline 3 & 0.170431 & 0.029519 & 0.007168 & 0.002037 & \\
\hline 4 & 0.118925 & 0.021702 & 0.005493 & & \\
\hline 5 & 0.091148 & 0.017114 & & & \\
\hline 6 & 0.073836 & & & & \\
\hline
\end{tabular}


Then the "free" parts of the first four lines of (4.18) evaluate to

$$
\frac{-4 \delta_{r s} \delta_{j k}}{B^{2}}\left[2 I_{4,3}-4 I_{3,3}+2 I_{2,3}+2 I_{5,2}-3 I_{4,2}+I_{3,2}\right] \approx \frac{-0.005436 \delta_{r s} \delta_{j k}}{B^{2}} .
$$

The 5th and 8th matrix elements are also equal, and their free contributions to (4.18) are easily shown to give

$$
\frac{-4 \delta_{r s} \delta_{j k}}{B^{2}}\left[I_{6,1}-2 I_{5,1}+I_{4,1}\right] \approx \frac{-0.04186 \delta_{r s} \delta_{j k}}{B^{2}}
$$

The 6th and 7th matrix elements in group 1 are the most tedious to evaluate, but the steps are straightforward, with the result for their free contribution to (4.18)

$$
\begin{array}{r}
\frac{-2 \delta_{r s} \delta_{j k}}{B^{2}}\left[2 I_{2,5}-4 I_{1,5}+2 I_{0,5}+4 I_{3,4}-6 I_{2,4}+2 I_{1,4}+2 I_{4,3}-2 I_{3,3}+I_{2,3}\right] \approx \\
\frac{-0.014932 \delta_{r s} \delta_{j k}}{B^{2}}
\end{array}
$$

Finally the free contribution to (4.19) is the same for each of the 4 matrix elements, leading to a total contribution

$$
\frac{-4 \delta_{r s} \delta_{j k}}{B^{2}}\left[-6 I_{6,1}+10 I_{5,1}-4 I_{4,1}\right] \approx \frac{+0.028944 \delta_{r s} \delta_{j k}}{B^{2}}
$$

We record here the total contribution from the "free" term of the Green function to (4.18) and (4.19), the sum of (A.4), (A.5), (A.6), and (A.7), -0.033284/B2, which is approximately $10 \%$ of the lowest order (RPA) term.

Next we turn to the contribution from the second term of (A.1) to the various 
terms in (4.18). The evaluation requires the matrix elements

$$
\begin{aligned}
\left\langle t+B\left|y^{j} \frac{1}{\mathbf{p}^{2}+B+t} p^{m}\right| B\right\rangle & =\frac{i B \delta_{j m}}{\pi} \int_{\infty}^{\infty} d \mathbf{p} \frac{\mathbf{p}^{2}}{\left(\mathbf{p}^{2}+B\right)\left(\mathbf{p}^{2}+B+t\right)^{3}} \\
& =\frac{i \delta_{j m}}{B} \int_{1}^{\infty} d u \frac{u-1}{u(u+t / B)^{3}}, \\
\left\langle t+B\left|p^{m} \frac{1}{\mathbf{p}^{2}+B} y^{j}\right| B\right\rangle & =\frac{-i B \delta_{j m}}{\pi} \int_{1}^{\infty} d \mathbf{p} \frac{\mathbf{p}^{2}}{\left(\mathbf{p}^{2}+B\right)^{3}\left(\mathbf{p}^{2}+B+t\right)} \\
& =\frac{-i \delta_{j m}}{B} \int_{1}^{\infty} d u \frac{u-1}{u^{3}(u+t / B)},
\end{aligned}
$$

and their complex conjugates. The integrals here are elementary:

$$
\begin{aligned}
& \int_{1}^{\infty} d u \frac{u-1}{u(u+v)^{3}}=\frac{1}{v(v+1)}\left(\frac{1}{v}+\frac{1}{2}-\frac{1}{v}\left(1+\frac{1}{v}\right) \ln (1+v)\right) \\
& \int_{1}^{\infty} d u \frac{u-1}{u^{3}(u+v)}=\frac{1}{v}\left(\frac{1}{v}+\frac{1}{2}-\frac{1}{v}\left(1+\frac{1}{v}\right) \ln (1+v)\right) .
\end{aligned}
$$

Using these results we find that the second term of (A.1) inserted into (4.18) yields

$$
\frac{4 \delta_{r s} \delta_{j k}}{B^{2}} \int_{1}^{\infty} d t \frac{t-1}{t^{4}(1+t) \ln (1+t)}\left(\frac{1}{t}+\frac{1}{2}-\frac{1}{t}\left(1+\frac{1}{t}\right) \ln (1+t)\right)^{2} \approx \frac{0.005795 \delta_{r s} \delta_{j k}}{B^{2}}
$$

for the first 4 lines of (4.18). For the 5th and 8th lines of (4.18) the corresponding contribution is

$$
\frac{-2 \delta_{r s} \delta_{j k}}{B^{2}} \int_{1}^{\infty} d t \frac{t-1}{t^{4} \ln (1+t)}\left(\frac{1}{t}+\frac{1}{2}-\frac{1}{t}\left(1+\frac{1}{t}\right) \ln (1+t)\right)^{2} \approx \frac{-0.009937 \delta_{r s} \delta_{j k}}{B^{2}}
$$


and for the 6th and 7th lines,

$$
\begin{array}{r}
\frac{-2 \delta_{r s} \delta_{j k}}{B^{2}} \int_{1}^{\infty} d t \frac{t-1}{t^{4}(1+t)^{2} \ln (1+t)}\left(\frac{1}{t}+\frac{1}{2}-\frac{1}{t}\left(1+\frac{1}{t}\right) \ln (1+t)\right)^{2} \approx \\
\frac{-0.000967 \delta_{r s} \delta_{j k}}{B^{2}} .
\end{array}
$$

The numerical values for these integrals were obtained by a simple integration routine. For reference purposes we also quote the values of the terms in (4.11) that have been cancelled, so that the total value of the first diagram in Fig. 3 can be worked out. This works out to a total of $-\delta_{r s} \delta_{k j} /\left(18 B^{2}\right)$. Noting that (A.8) almost cancels (A.4), we see that this last bit is the dominant contribution to the diagram, about $16 \%$ of the RPA contribution.

The contribution from the last three terms of (A.2) to (4.19) requires the matrix elements

$$
\begin{aligned}
\left\langle B\left|y^{j} \frac{1}{\mathbf{p}^{2}+B} y^{k}\right| B\right\rangle & =\frac{\delta_{j k}}{6 B^{2}} \\
\left\langle B^{\prime}\left|y^{j} \frac{1}{\mathbf{p}^{2}+B} y^{k}\right| B\right\rangle & =-\frac{\delta_{j k}}{5 B^{2}} \\
\left\langle B\left|p^{m} \frac{1}{\mathbf{p}^{2}+B+t} p^{m}\right| B\right\rangle & =\int_{1}^{\infty} d u \frac{u-1}{u^{2}(u+t / B)} \\
\left\langle B^{\prime}\left|p^{m} \frac{1}{\mathbf{p}^{2}+B+t} p^{m}\right| B\right\rangle & =-\frac{1}{B} \int_{1}^{\infty} d u \frac{u-1}{u^{3}(u+t / B)} .
\end{aligned}
$$

Using these results, we then obtain for the remainder of the evaluation of (4.19)

$$
\begin{aligned}
\frac{-4 \delta_{r s} \delta_{j k}}{B^{2}}\left[\left(\frac{1}{12}-\frac{1}{5}\right)\left(I_{1,1}-I_{2,1}\right)-\frac{1}{6}\left(I_{2,1}-I_{3,1}\right)\right] & = \\
& \frac{\delta_{r s} \delta_{j k}}{B^{2}}\left[\frac{7}{15}\left(I_{1,1}-I_{2,1}\right)+\frac{2}{3}\left(I_{2,1}-I_{3,1}\right)\right] \approx \frac{0.359069}{B^{2}}
\end{aligned}
$$

Finally, we evaluate the double pole contributions (4.16) and (4.16), which requires 
the additional matrix elements

$$
\begin{aligned}
\left\langle B\left|y^{j} \frac{1}{\left(\mathbf{p}^{2}+B\right)^{2}} y^{k}\right| B\right\rangle & =\frac{\delta_{j k}}{10 B^{3}} \\
\left\langle B\left|p^{m} \frac{1}{\left(\mathbf{p}^{2}+B+t\right)^{2}} p^{m}\right| B\right\rangle & =\frac{1}{B} \int_{1}^{\infty} d u \frac{u-1}{u^{2}(u+t / B)^{2}} .
\end{aligned}
$$

Then the total double pole contribution evaluates to

$$
\frac{\delta_{r s} \delta_{j k}}{B^{2}}\left[\frac{1}{5}\left(I_{1,1}-I_{2,1}\right)+\frac{1}{3}\left(I_{1,2}-I_{2,2}\right)\right] \approx \frac{0.133316}{B^{2}}
$$

The grand total of all contributions is

$$
\text { TOTAL } \approx \frac{0.453992 \delta_{r s} \delta_{k j}}{B^{2}}
$$

which is $-136 \%$ of the RPA result. Clearly this cannot be regarded as a small correction!

\section{REFERENCES}

1. R. Giles and C. B. Thorn, Phys. Rev. D16 (1977) 366.

2. C. B. Thorn, Phys. Rev. D17 (1978) 1073.

3. C. B. Thorn, Phys. Rev. D19 (1979) 639. See also I. Klebanov and L. Susskind, Nucl. Phys. B309 (1988) 175, for a lattice model with similar physics.

4. V. A. Kazakov, Mod. Phys. Lett. A4 (1989) 2125.

5. V. A. Kazakov and A. A. Migdal, Nucl. Phys. B311 (1988/89) 171.

6. E. Brezin and V. A. Kazakov, Phys. Lett. 236B (1990) 144.

7. D. J. Gross and A. A. Migdal, Phys. Rev. Lett. 64 (1990) 127; Phys. Rev. Lett. 64 (1990) 717; Nucl. Phys. B340 (1990) 333. Phys. Rev. Lett. 64 (1990) 717. 
8. M. R. Douglas and S. H. Shenker, Nucl. Phys. B335 (1990) 635.

9. C. B. Thorn, "Reformulating String Theory with the 1/N Expansion," in Sakharov Memorial Lectures in Physics, Ed. L. V. Keldysh and V. Ya. Fainberg, Nova Science Publishers Inc., Commack, New York, 1992. hepth/9405069

10. J. Goldstone, Proc. Roy. Soc. (London) A239 (1957) 267.

11. P. Goddard, J. Goldstone, C. Rebbi, and C. B. Thorn, Nucl. Phys. B56 (1973) 109.

12. G. 't Hooft, Nucl. Phys. B72 (1974) 461.

13. C. B. Thorn, Phys. Rev. D20 (1979) 1435.

14. J. Goldstone, private communication (1978)

15. D. J. Gross and P. F. Mende, Phys. Lett. 197B (1987) 129; Nucl. Phys. B303 (1988) 407.

16. G. 't Hooft, Nucl. Phys. B342 (1990) 471; "On the Quantization of Space and Time," Proc. of the 4th Seminar on Quantum Gravity, 25-29 May 1987, Moscow, USSR, ed. M. A. Markov et al., (World Scientific Press, 1988); "Dimensional Reduction in Quantum Gravity," Utrecht preprint, THU-93/26, gr-qc/9310026.

17. L. Susskind, L. Thorlacius, and J. Uglum, Phys. Rev. D48 (1993) 3743.

18. L. Susskind, "Some Speculations About Black Hole Entropy in String Theory," Rutgers Univ. preprint RU-93-44, hep-th/9309145; L. Susskind and J. Uglum, "Black Hole Entropy in Canonical Quantum Gravity and Superstring Theory," Stanford Univ. preprint, 